\title{
Gradhiva
}

GRADHIV

Revue d'anthropologie et d'histoire des arts

32 | 2021

Livres sorciers

\section{Écritures nocturnes. Régénération et circulation des écrits chez les Mayas cruzo'ob}

Nightly Texts. The Regeneration and Circulation of Writing among the Cruzo'ob Maya

Valentina Vapnarsky et Hilario Chi Canul

\section{CpenEdition}

Journals

Édition électronique

URL : https://journals.openedition.org/gradhiva/5469

DOI : 10.4000/gradhiva.5469

ISSN : $1760-849 x$

Éditeur

Musée du quai Branly Jacques Chirac

Édition imprimée

Date de publication : 24 mars 2021

Pagination : 82-105

ISBN : 978-2-35744-132-3

ISSN : 0764-8928

Référence électronique

Valentina Vapnarsky et Hilario Chi Canul, «Écritures nocturnes. Régénération et circulation des écrits chez les Mayas cruzo'ob », Gradhiva [En ligne], 32 | 2021, mis en ligne le 02 avril 2021, consulté le 25 mai 2021. URL : http://journals.openedition.org/gradhiva/5469; DOI : https://doi.org/10.4000/ gradhiva.5469

(c) musée du quai Branly 
$\therefore$ Nun

nomegund yosassitur pes sim gutos dies fivican vessi fruesti tis gla dium sum Vi brabito as livin Siuntetern dit so pa

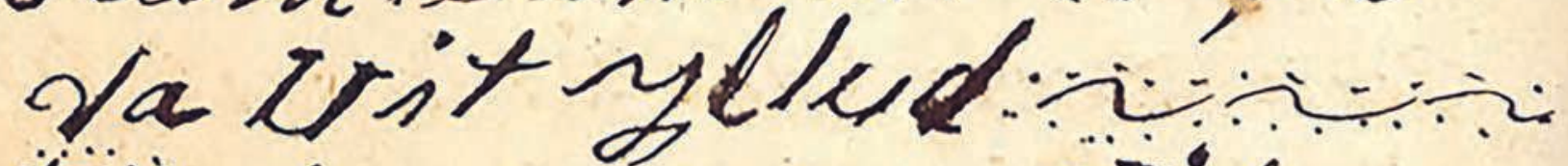
Fifi in loparabit ia samor tis tass vas asben ti bus effifie cit fipice par to s diitin pis It tian Can Ce sf ist Do lowem pepesit ingui taten iacma pe serit \& e ifroditem en sos in $C_{i}$ dit in fio trean mann guasan frecitivi: 
Écritures nocturnes

Régénération

et circulation

des écrits chez

les Mayas cruzo’ob 
À partir de la découverte récente de carnets manuscrits mayas, vieux de plus d'un siècle, retrouvés chez un sorcier bibliomane, qualifiés d'écriture nocturne et reliés à des généalogies d'écrits sacrés et subversifs, cet article s'attache à élucider les différents sens que revêtent ces écrits et l'écriture dans la société maya des descendants des Mayas cruzo'ob de la guerre des Castes au Mexique. L'article débute par un récit personnel d'Hilario Chi Canul, à qui furent remis ces écrits. Le récit, originellement rédigé dans sa langue maternelle, le maya yucatèque, est traduit et annoté en français par Valentina Vapnarsky. Celle-ci poursuit, assumant le « je » des parties restantes, alors que le « nous » témoigne de la collaboration menée depuis quatre ans entre les deux coauteurs ${ }^{1}$.

1. Nous remercions Pierre Déléage pour les suggestions apportées à une première version de cet article.

2. «Grand frère» traduit suku'un, un terme de parenté mais aussi d'adresse marquant le respect. L'équivalent pour une femme est $k$ iik, traduit par «grande sœur» plus loin dans le texte.

\section{DE VIEUX ÉCRITS MAYAS ET LEUR CHIEN NOIR}

«J'ai un écrit ancien chez moi, quand tu rentreras au Mexique, ce serait bien que tu passes le voir, tu verras si tu y comprends quelque chose. On me dit que c'est en maya, mais moi, même si je le parle, je n'y comprends rien! Je crois que c'est du maya très très ancien, parce que quand je le lis, c'est comme si ce n'était pas du maya. Je n'y comprends vraiment rien, mon garçon!»

Voilà ce que me dit celui qui était alors maire de Noj Kaaj Santa Kruus x Báalam Naj K'anpok'olche' Kaaj (dans l'état du Quintana Roo, au Mexique), quand il vint me voir en Bolivie pour la cérémonie de remise de mon diplôme de master. C'était en 2011. Je lui répondis :

"Qu'est-ce que ça peut bien être comme document? Où l'avez-vous pris? Et pourquoi dites-vous que c'est du maya, mais que ça n'y ressemble pas?»

Après avoir entendu mes paroles, grand frère ${ }^{2}$ le maire, qui était en train d'écrire sur son téléphone portable, leva les yeux. Seul son index resta planté sur l'écran. Il me regarda et me dit:

«Garçon, ce dont je te parle appartenait à un jmeen [chamane], je l'ai trouvé dans une caisse qui était restée dans une maison abandonnée que j'ai achetée, dans un petit village au nord de Carrillo Puerto. Si tu savais, la maison, elle a brûlé, mais pas le document.»

Il ôta son index du téléphone portable, le posa sur ma poitrine, puis ajouta:

«Quand tu reviendras, passe le prendre, tu verras s'il peut te servir, si tu le comprends! Sinon, je le jetterai. Comme toi tu lis le maya, il est possible que tu y comprennes quelque chose. Moi, je n'y comprends goutte, ça ne me sert à rien. Ce document, son maître était un jmeen, et on dit que de son vivant, c'était même un sorcier très puissant!

- D'accord, je passerai le prendre chez vous quand je serai rentré», assurai-je.

Une année et quatre mois passèrent jusqu'au jour où l'ancien écrit me parvint: grand frère le maire me le remit en main propre, chez lui, à midi, le samedi 8 du mois de janvier de l'année 2012. Je me souviens du message écrit qu'il m'envoya sur mon téléphone portable, ce même matin, et qui disait [en espaǵnol] :

«Amigo, vas-tu passer chercher le livre dont je t'ai parlé? Aujourd'hui, je serai à la maison à midi. »

Je lui répondis [en espaǵnol] :

«Bien sûr, amigo, je vous verrai chez vous à midi.»

À l'heure dite, donc, j'étais à la porte de la maison de ce grand frère en possession de l'écrit.

[...] Il me fit rentrer chez lui et nous nous assîmes autour d'une table. Il y posa le manuscrit en annonçant:

«Hermano, voici ce dont je t'avais parlé. J'ai interrogé l'homme qui a sauvé les papiers de la maison brûlée, comme je te l'ai raconté. Il paraît qu'on les appelait des áak'ab tsíib ["écritures nocturnes"], parce que le jmeen à qui ils appartenaient autrefois, on dit que c'est seulement de nuit qu'il les lisait; de jour, prétendon, les lettres n'étaient pas visibles. Voici, je les remets entre tes mains. Comme je te l'ai dit, tu es le premier à qui j'ai parlé de ces écrits. Emporte-les, étudie-les, puis tu me les rendras. De mon côté, je les protégerai du mieux possible.»

Sur ces paroles, grand frère le maire me tendit les écrits anciens, enveloppés dans un sac en plastique jaune. Je ne dis rien mais j'opinai du chef à plusieurs reprises, en signe d'accord. Puis, je pris le paquet 
Exurge Domine imi ir a twa: he sallaye im firm bie Si mi mi corum meonum (bit ¿ $^{x}$ ux ge Domine Deus meus impre ceb to giod manda sti \& \& $S_{y}$ no coga populo reum cir cum da bibi te. Ex propter hamci mal tum re gre de re Domimus Ticdicat popu lus Tu dicame Do-nine Se cumdum Tus titiam is: Is Se cunhdumimnocen tiam meam Su per me Wan Su ne tuphe quitia pee calarum te diziges Jus tum kize nes geus di Jus tum ad putorium meum a Domi no guisal Llo S Facit ree dos corde we gus.u dex justus por tis \& patie ns solsi sican orer si
Pueritis gla qium Sum Nivibabito arcurt Sumterm dit h para ctir yllud stimlopsparit Lta Sa mar tins:is git tass thas as dem ti bus

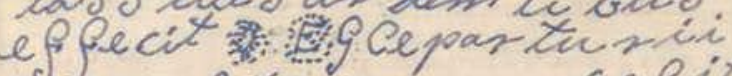
in jus ti tiam can Ce frit Dolorem \& pepar ix imi guitaten sacunapl pulit \& $e$ fifiodit ecim qincidit dimfo $86 a m$ quo mian frecix sitomilter te tur Dollor e puis im ca puteyus is te im Iter ticemipsius im -quitas e pus deciem det Cosonfitebo - Domine Se cumdum jus titiam e jus k p $S$ - allamo iniimi Dosi - ma ni al tis Si mi

Pages d'un des carnets de prière mayas étudiés [c1, 16-17], région cruzo'ob, Mexique, début du xxe siècle. 
3. Selon les Mayas yucatèques, sur terre circulent de très nombreux «airs》 attachés à des lieux ou à des entités diverses et pouvant, sous des conditions variées, «attraper» le corps des personnes, produisant divers effets pathogènes. Ici, il s'agit plus spécifiquement d'un air de type saalat, un air léger (sáal) qui pénètre lentement.

4. L'expression maya employée pour décrire l'écriture cursive, tsa'ayla'an ts'íib, signifie presque littéralement «écriture attachée»: tsa'ayla'an est un participe construit sur la racine TSAY, «unir, joindre, associer une chose à la suite d'une autre», et $t s^{\prime} i i b$, «écrire, tracer/ écriture». Le terme ts'íib est très ancien, il était déjà utilise désigner l'écriture glyphique, au côté de wooj. Seul le premier a perduré de façon productive pour désigner l'écriture alphabétique, souvent joint à l'époque coloniale au mot pour pour «papiernju'un (Hanks 2010; Cunill 2020). Depuis la fin du $x x^{\mathrm{e}}$ siècle, I'al phabétisation dans les écoles de la région maya, et du Mexique plus généralement, se fai uniquement en caractères d'imprimerie, cequi a res d'imprimerie, ce qui a entraîné
une survalorisation de l'écriture en lettres attachées, considérée comme plus ancienne, plus difficile et plus ésotérique.

5. Le chicle est le latex du sapotier. Son extraction et son commerce furent, avec la vente de bois, la principale source

de revenus des Mayas cruzo'ob au début du $x x^{e}$ siècle. qu'il avait déposé devant lui, sans le défaire. Je sentis qu'il était épais, ma main pouvait à peine le saisir. Alors je déclarai :

«Grand frère, je te remercie vivement pour ta confiance. Quand viendra le jour de te le rendre, je t'expliquerai tout ce que j'en ai compris.

- C'est bien ainsi, hermano. Je t'enverrai aussi le numéro de téléphone d'un ami qui connaît très bien les anciens du villaǵe d'où vient le document. Je lui ai dit de t'emmener à Tusik' pour que tu y rencontres un des jmeen de là-bas.

- D'accord, grand frère. Quand le numéro de cet homme m'arrivera, je l'appellerai pour que nous allions à Tusik'. Je pense que les grands hommes de là-bas me seront d'une grande aide pour comprendre cette écriture ancienne.»

Je me levai alors, lui serrai la main et pris congé. Puis je quittai sa maison.

Je marchais sur le chemin de retour quand il se mit à bruiner. Je ǵlissai vite le paquet sous mon bras pour le protéger et accélérai le pas afin d'arriver avant que les papiers ne se mouillent. Mais soudain je fus pris d'un frisson, mes cheveux et mes poils se dressèrent, mon corps tout entier se mit à trembler, car la pluie qui ruisselait sur mon corps était très froide très froid aussi le vent qui l'accompagnait. J'arrivai prestement chez moi, où, une fois les mains sèches, je me jetai dans un hamac avec le paquet pour l'essuyer. En commençant à le dénouer, j'aperçus le tissu qui couvrait l'un des carnets. Aussitôt je sentis disparaître le froid qui m'avait saisi, il me sembla qu'un air saalat chaud me traversait le corps depuis la cheville jusqu'à la nuque, d'où je le sentis sortir ${ }^{3}$. Je retirai le premier cahier écrit du paquet, celui à la couverture en tissu; je compris qu'il était sur le point de se défaire car les pages, quand je les tournais, restaient suspendues au fil de la reliure. Quand je l'ouvris, je vis immédiatement qu'il contenait des textes en lettres attachées ${ }^{4}$ et que en effet, c'était de l'écriture maya. La première ligne indiquait qu'il s'aǵissait «du commencement des Macehual» (autodésignation des Mayas de la région). Mon regard parcourut la page jusqu'en bas, puis je la tournai et vis à nouveau que le texte était en maya authentique (jach maaya), j'en lisais les passages que je comprenais, puis je passais à une autre page. Je feuilletai le cahier jusqu'à me trouver face à des pages sans écriture, toutes blanches, sauf à certains endroits, où figuraient quelques comptes, noms et l'inscription de ventes ou d'achats de chicle 5 .

Je refermai alors ce cahier et en tirai un autre de la liasse. Le dessus de ce dernier était plus sale, on aurait dit que sa couverture avait été arrachée. Il était évident qu'il avait aussi été recouvert d'un tissu collé, car il en restait des traces ici et là. Avant même d'en tourner les pages, je reconnus des écritures; ce cahier commençait par de nouvelles inscriptions de ventes ou d'achats; j'avançai et arrivai à d'autres textes en lettres attachées; ceux-là, je ne les comprenais pas. La troisième liǵne commençait par «Regen », entièrement encerclé de petits points; ce qu'il y avait à côté et en dessous, je ne le saisis pas. Je me mis à en tourner les paǵes jusqu'à d'autres écritures, de couleur bleuâtre, mais ne les compris pas non plus. Je remarquai simplement les petits points tout autour, c'était très beau la façon dont ils enveloppaient le corps de l'écriture, certains étaient comme de petits vers de terre couverts d'épines en train de se déplacer, d'autres ressemblaient à des mille-pattes, et d'autres encore prenaient la forme de petits papillons aux ailes déployées, bordées de franges de fils fins, ou de nids de minuscules moucherons. Je continuai done, je sortis d'autres cahiers, vis qu'ils contenaient des calliǵraphies et des couleurs très variées, dont le corps et le contenu différaient également: l'écriture était tantôt noire, tantôt gris charbon ou bleue, parfois d'un cendré foncé; certains textes étaient très lisibles, alors que d'autres étaient très obscurs. Je constatai que, oui, ces écrits étaient vraiment anciens. Il y avait peut-être une dizaine de cahiers, en fait, je ne compris pas combien précisément car certains étaient comme collés les uns aux autres. Alors, je les empilai de nouveau, les remis dans le sac en plastique jaune et cherchai une petite boîte en carton où je pourrais les conserver, tels qu'ils avaient été trouvés.

Cette nuit-là, je fis un terrible cauchemar. Il était peut-être un peu plus de minuit quand je me couchai dans mon hamac bleu foncé. À peine avais-je fermé les yeux que je m'endormis et, immédiatement, je fis un rêve: un grand chien noir m'apparut à l'ouest. Je parlais, mais les paroles ne sortaient pas. J'étais tétanisé de peur à la vue de ce ǵros chien s'approchant de moi à grands pas, vers la pointe de mes pieds. Je regardai autour de moi : ma mère dormait, ma compagne dormait aussi, ainsi que ma petite fille, j'étais le seul éveillé. Je les appelais, mais personne n'entendait ma voix car les paroles ne sortaient pas de ma bouche, je la sentais toute crispée. Alors, je me souvins que feu mon père m'avait enseigné que si de mauvaises choses venaient à traverser mon chemin, il me faudrait, pour les surmonter, réciter en boucle le credo. Donc, comme ma voix ne sortait pas, je me mis à réciter le credo tout en observant le grand chien s'avancer encore vers moi. Il atteiǵnit la pointe de mes pieds. Il avait la gueule grande ouverte, sa bave coulait, il se tenait debout face à moi, harǵneux. Il était très grand, sa poitrine arrivait à la hauteur du bord de mon hamac, son poil brillait, son pelage noir uniforme le couvrait tout entier et sa gueule était rouge vif maculé de noir. Moi, je priais dans ma tête, je priais pour le maîtriser et pouvoir me réveiller. Alors, le chien cessa de me regarder, pivota vers le sud et se remit en marche. Je le vis arriver à la hauteur de ma tête, puis me tourner autour. Il ne passa pas sous mon hamac, il était vraiment immense. Je le sentis arriver droit sur ma tête, et soudainement, je me réveillai. Je m'assis dans mon hamac. J'avais peur! J'attrapai mon téléphone par terre, en allumai la lampe torche, puis j'appelai ma mère (qui dormait dans le hamac voisin) :

«Maman, maman, maman!

- Qu'y a-t-il? Qu'est-ce qu'il se passe? me dit-elle.

- J'ai fait un terrible cauchemar, lui répondis-je.

- Lève-toi ! Allume la lumière!» me dit-elle. 


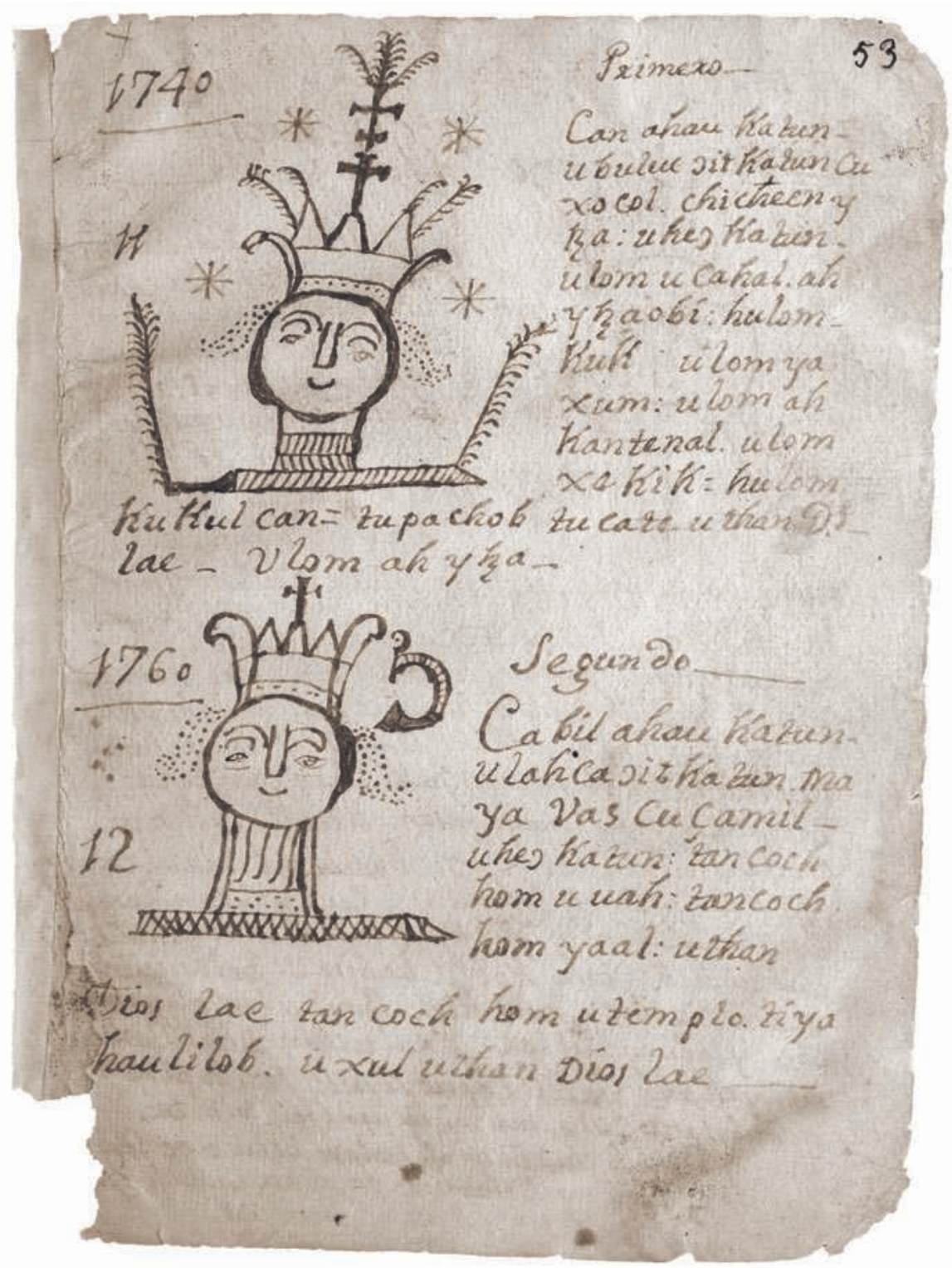

Livre du Chilam Balam de Chumayel, 1775-1800, Yucatán, Mexique. Papier. Manuscrit écrit langue maya, $20 \times 15 \mathrm{~cm}$.
Princeton Mesoamerican Manuscripts n 4, Manuscripts Division, Department of Rare Books and Special Collections, Princeton University Library,
C9040, p. 103.

Ce que je fis. Elle ajouta:

«Prie! Sans t'en rendre compte, tu as dû dormir avec ton bras gauche sur la poitrine, ce doit juste être ça. Je crois qu'il va bientôt faire jour.»

Sur mon portable, je vis qu'en effet il était déjà presque 4 heures du matin. Je me remis à prier et me rendormis. Cette fois, je n'éteiǵnis pas la lumière et surtout, je fis attention à bien laisser mon bras replié sur le côté, de façon à ne pas replacer ma main gauche sur la poitrine.

Le jour arriva, nous nous levâmes pour petitdéjeuner. Avant toute chose, je racontai mon rêve à ma mère: je lui dis comment j'avais vu le chien noir et l'avais dominé avec mes prières. Après m'avoir écouté, ma mère me demanda:

«Es-tu allé quelque part ou as-tu fait quelque chose de spécial hier?»
Je rétorquai :

«Eh bien, non, rien, je suis simplement allé chercher les cahiers, comme je vous l'avais dit, je n'ai été nulle part ailleurs.»

Elle me dit :

«Quels cahiers?

- Eh bien, les cahiers d'écrits anciens que je suis allé chercher à la maison de l'ex-maire.

- Ah, c'est donc ça dont tu as rêvé. Les choses anciennes, elles ont leur "air". C'est parce que tu les as manipulées que leur maître est venu à toi. »

Je répliquai :

«Mais comment vais-je faire pour les lire alors? Ils m'ont été donnés pour que je les lise, et c'est ce que j'ai voulu faire à peine rentré. Mais c'est vrai que, dès que j'ai ouvert le sac, j'ai tout de suite senti un 


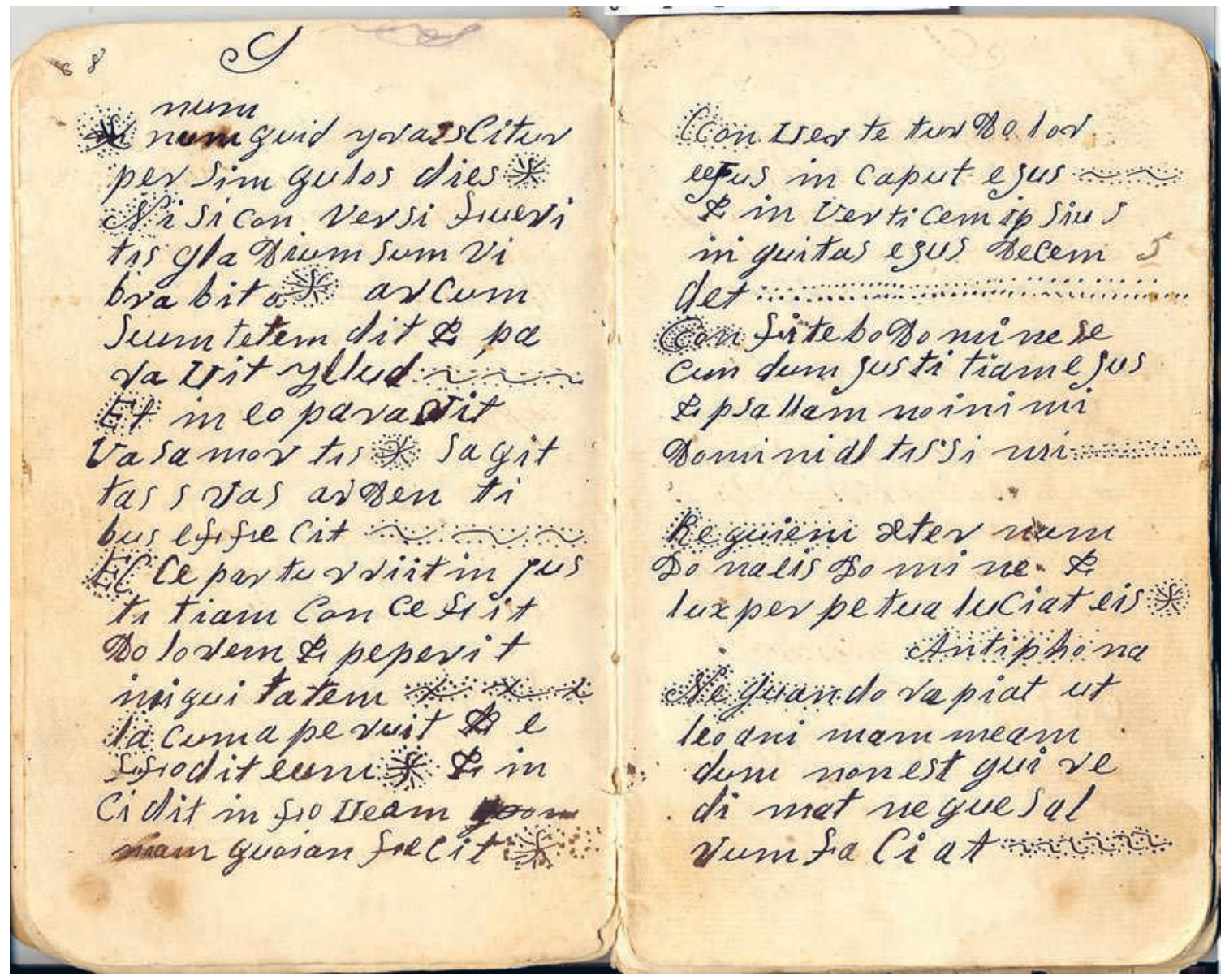

Pages d'un des carnets de prière mayas étudiés [c2,10-11], région cruzo'ob, Mexique, début du xxe siècle.

6. Diminutif de Valentina ( $x$ marque le féminin).

7. «Grand homme» traduit ici nojoch máak, terme respectueux de référence et d'adresse pour les adultes. air chaud me traverser le corps. J'ai cru qu'il s'agissait simplement du froid qui m'avait pris sur le chemin de retour, quand la bruine m'avait mouillé.

- Non, fils, répondit ma mère, ça, c'est l'air de ces vieilles choses. Pour pouvoir toucher ces papiers, tu dois faire un dépôt d'offrandes, demander la permission de les lire et balayer leur mauvais air pour qu'ils ne te fassent pas de mal. C'est ce que ma mère me dit encore.

- Eh bien, je vais faire ce dépôt d'offrandes dès aujourd'hui, car je ne veux vraiment pas en rêver de nouveau. Et puis, il faut que je lise les textes.

- Tu fais bien. En fait, ce ne sera pas un gros travail, il suffira que tu tues une volaille; et nous nous agenouillerons pour la remettre en offrande. »

C'est done ce que je fis. Cela me permit de reprendre les anciens écrits et de commencer à les étudier avec d'autres compaǵnons, certains même venus de pays étrangers, dont la France. Depuis ce jour-là, je n'ai plus jamais rêvé de choses aussi terrifiantes que cette nuit-là. Mais c'est certain, nous savons maintenant que celui qui avait ces écrits anciens en sa possession était vraiment un grand sorcier. Cela, je l'ai découvert quand, afin de comprendre les écrits des cahiers, je suis allé jusqu'au village de Tusik'. L'homme qui devait m'y conduire m'a raconté qu'il avait connu le possesseur des écrits anciens que j'avais désormais entre les mains. Il me raconta que le vieil homme se transformait en un grand chien noir. Au village, quand il marchait sur le chemin qui conduisait à son champ, tu voyais avancer un grand chien noir, c'était lui. Si tu le croisais et lui parlais, il se dressait et se transformait à nouveau en homme. Et puis, on disait que c'était un homme très colérique; si tu faisais quelque chose qui ne lui plaisait pas, il s'arrangeait pour que tu te souviennes de lui ! C'est pourquoi chacun faisait très attention en s'adressant à lui. C'est aussi ce que l'on nous dit dans un autre village au nord de Carrillo, quand nous nous y sommes rendus avec grande sœur xBalen ${ }^{6}$ pour comprendre les textes. Un ancien de là-bas, qui nous entendit mentionner celui qui possédait ces vieux écrits, nous recommanda immédiatement de faire très attention si nous en venions à évoquer cet homme:

«Écoutez ce que je vais vous raconter, dit-il, ce que j'ai vu, de mes yeux vu: une fois, j'étais parti rendre visite au grand homme ${ }^{7}$, j'étais chez lui quand passa un petit homme qui allait vendre une vache dans un village voisin. Le pauvre homme passa done devant la maison de mon hôte, mais il omit de le saluer. Alors l'homme dit: "Voici donc comme il me traite, il ne me salue pas alors qu'il passe devant ma maison! Tu vas voir maintenant comment je vais le lui rendre." Il arracha aussitôt un bout des palmes de son toit, le frotta entre ses mains, puis souffla dessus; au même instant, une mauvaise chose fila au sol. Il lui dit: "Dépêche-toi de courir après ce fainéant, fais-le revenir sur ses pas pour me regarder en face." Et il lança le serpent, qui fila. Et ne voici pas que peu après, le pauvre homme revenait en marchant péniblement. "Grand homme, 
Écritures nocturnes, régénération et circulation des écrits chez les Mayas cruzo'ob

Valentina Vapnarsky et Hilario Chi Canul

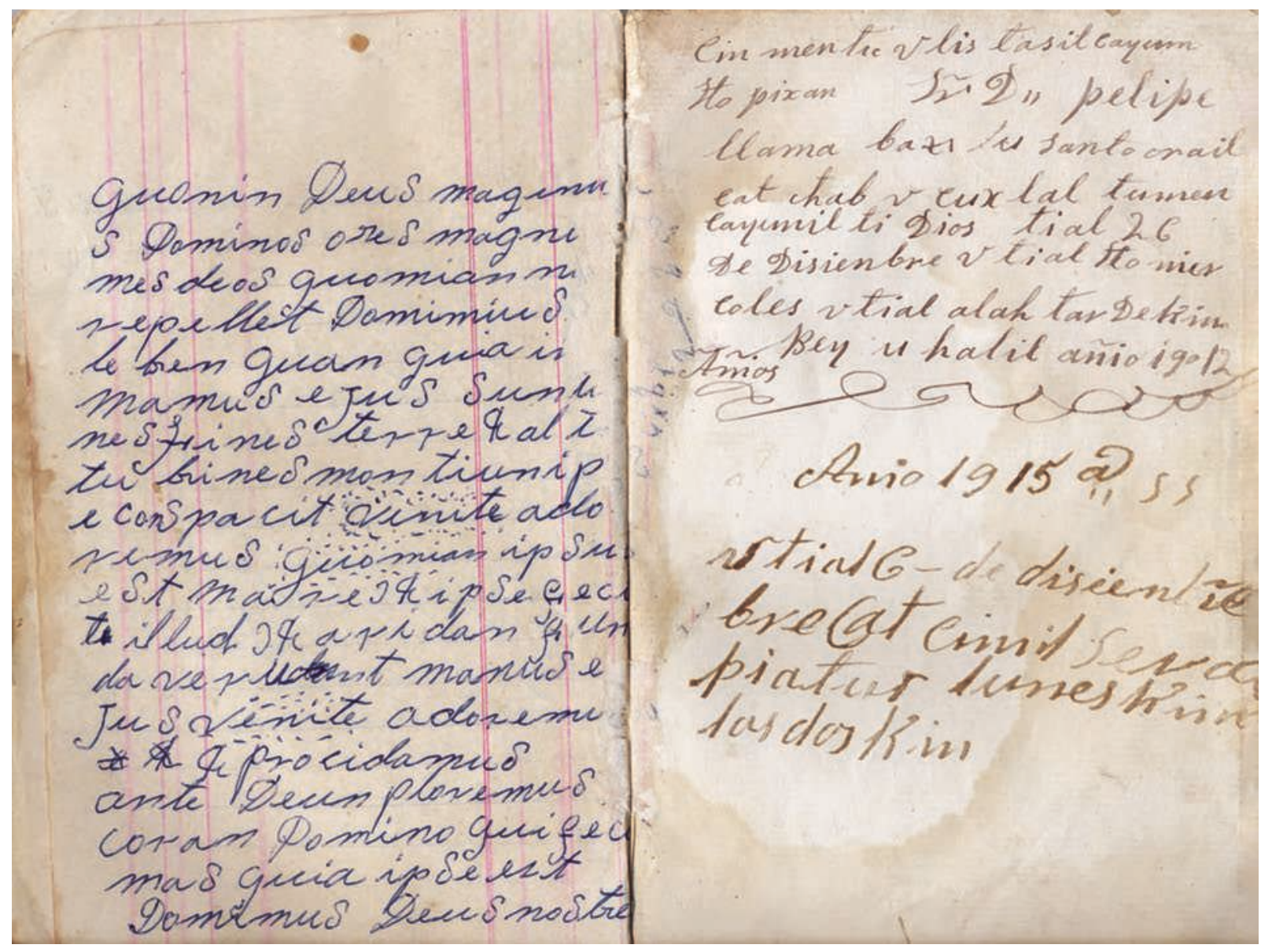

Pages de deux carnets ayant été joints entre eux, la page de droite inclut des références à des décès datés de 1912 et1915 [c3, 34-35], région cruzo'ob, Mexique, début du xxe siècle.

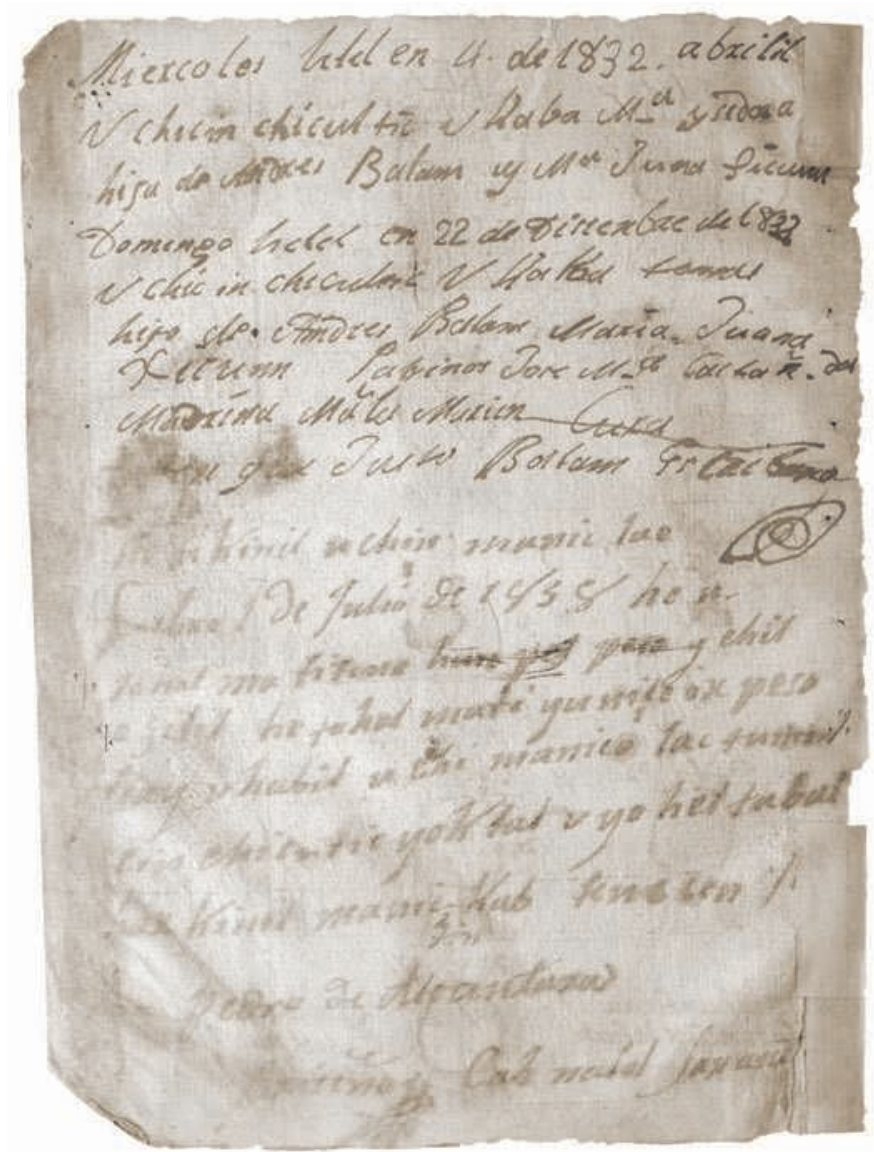

Livre du Chilam Balam de Chumayel, 1775-1800, Yucatán, Mexique. Papier. Manuscrit écrit langue maya, $20 \times 15 \mathrm{~cm}$. 
8. Du hip hop à Whatsapp: Nouvelles formes de communication chez les Amérindiens, 31 mars 2016, ANR Fabriq'Am, Paris. grand homme, lui dit-il, la mauvaise chose m'a fait du mal [= le serpent m'a piqué]." Mon hôte ne lui répondit pas, il passa simplement son bras derrière son dos, arracha une petite plante et la frotta entre ses mains. Puis il cracha dessus, et plaça cette mixture sur la jambe du petit homme. C'est tout ce qu'il fit, et il l'a soignné. Quand le pauvre homme fut parti, il me dit:

"Alors, qu'est-ce que t'en dis?." Comment pouvait-il ne pas le saluer? Ici personne ne passe sans le saluer.»

\section{TEXTES PRODIGIEUX ET ÉCRITURES DE GUERRE}

«Grande sœur, quand j'irai en France, je voudrais te montrer quelque chose qu'on m'a confié. Ce sont des écrits anciens. C'est en maya ancien. Je vois que c'est du maya mais je ne le comprends pas vraiment, enfin, seulement en partie. Je crois que c'est peut-être un Chilam Balam. Le Chilam de Noj Kaaj Sáanta Kruus x Báalam Naj K'anpok'olche' Kaaj, le nom du saint village y est écrit. Comme tu étudies le maya et les prières, tu pourras peut-être m'aider à comprendre.»

Voilà ce que m'écrivit Hilario peu de temps avant d'arriver à Paris où je l'avais invité à participer à un colloque sur les nouvelles formes d'expressions amérindiennes ${ }^{8}$. J'avais connu Hilario à peine un an auparavant, lors d'une autre rencontre où il m'avait entendue faire une présentation sur l'analyse sonore de discours rituels mayas. Il avait semblé particulièrement touché par mes enregistrements de Don Yano, un jmeen, spécialiste rituel réputé dont il avait entendu parler dans sa jeunesse. Nous avions découvert alors que nous avions vécu en voisins sans le savoir. Hilario était natif d'un village situé à une petite cinquantaine de kilomètres de celui où je m'étais installée plus de vingt ans auparavant pour mener des recherches dans la région.

Ces villages se trouvent à l'est de la péninsule du Yucatán, dans un territoire bien connu pour ses croix parlantes, ses Mayas rebelles et ses écrits de Juan de la Cruz. De tout temps zone de refuge des Mayas fuyant le pouvoir colonial, il avait été le berceau d'une nouvelle société maya fondée au milieu du XIX ${ }^{\mathrm{e}}$ siècle suite à une rébellion majeure (la "guerre des Castes», renommée récemment "Guerre sociale maya»). Les rebelles avaient fondé un premier hameau autour d'un cénote auprès duquel était apparue une croix miraculeuse ainsi qu'un écrit sacré (Vapnarsky 2017). Ce hameau avait ǵrandi et reçu le nom de Noj Kaaj Saanta Kruus x Báalam Naj K'anpok'olche' Kaaj (le nom qu'Hilario mentionnait et qu'il avait retrouvé dans les écrits). La croix avait assuré aux Mayas rebelles une communication directe avec Vrai Dieu ou Juan de la Cruz. Par ses pouvoirs, elle avait joué un rôle fondamental dans la poursuite de la guerre et des négociations avec leurs ennemis ou leurs alliés (Reed 1971, Dumond 1997; Sullivan 1989). Pour ces derniers et les historiens, elle était devenue la Croix Parlante, alors que les Mayas la considéraient comme la Santísima, la plus importante de leurs saanto (croix et figures de saints). Juan de la Cruz, figure christique et messianique, à la fois unique et multiple, s'exprimait à la première personne au travers de la croix et de ses interprètes, autant que par le biais de «testaments», de «jurements» et de lettres qu'il dictait miraculeusement aux «secrétaires de la croix » et qui se multiplièrent au fur et à mesure que la croix cessa de parler à voix haute et que s'intensifiaient les négociations diplomatiques des leaders mayas (Bricker 1981; Dumond 1997; Sullivan 1989).

Certains de ces écrits étaient des Almaj t'aan ou saanto ju'un, lus avec ferveur lors de cérémonies en l'honneur de la croix, auxquelles tous devaient assister. Les autres écrits étaient des lettres diplomatiques envoyées tantôt aux chefs de l'armée yucatèque, avec qui les rebelles entretinrent des relations belliqueuses jusqu'au début du $\mathrm{xx}^{\mathrm{e}}$ siècle, tantôt aux autorités britanniques installées au Belize, pour réǵler notamment les échanges de bois contre des armes. Plus tardivement, dans les années 1930, elles furent adressées au président et aux autorités des États-Unis via l'entremise de l'archéologue Sylvanus Morley et de l'anthropologue mexicain Alfonso Villa Rojas (Sullivan 1989). Tous les écrits de Juan de la Cruz, qu'il s'agisse des saanto ju'un ou de sa correspondance, déployaient sur un ton exalté une rhétorique catholique messianique, évoquant leur énonciateur, Juan de la Cruz, sous les motifs d'un Christ tourmenté par les souffrances de la Passion. Ils étaient assortis des meilleures figures parallélistiques de l'art verbal maya, des formes de la profération prophétique, ainsi que de la menace et de l'autorité martiales (Bricker 1981; Vapnarsky 2017 chap. IV, 2018).

Au fil des décennies, d'autres croix parlantes et de nouveaux saanto ju'un sont apparus, consacrant la fondation de nouveaux centres cérémoniels qui formèrent un ensemble de cinq. Chaque centre reproduisait l'orgánisation sociale, religieuse et militaire et la liturǵie des autres. La lecture des écrits de Juan de la Cruz en était une des clefs de voûte, surtout depuis que les croix avaient cessé de parler. La divinité ne s'adressait désormais plus directement à ses «âmes engendrées». D'abord, elle laissa des écrits, puis fit perpétuer sa mémoire grâce à des lectures publiques hautement ritualisées. Ces lectures devaient rassembler le plus grand nombre de Mayas, tout en restant occultes à toute personne extérieure au groupe. Aujourd'hui encore, certains disent que les lectures continueraient de se faire mais que seules les autorités mayas y assisteraient; d'autres qu'on ne les fait plus mais que les écrits sont encore là, religieusement cachés et protégés; d'autres que les écrits ont été vendus; d'autres encore qu'ils sont conservés mais que personne ne peut les lire. Lors des fêtes patronales, les Mayas éméchés continuent de provoquer régulièrement les étranǵers (non Mexicains particulièrement), en les incitant à prendre des photos (strictement interdites et qui vous mènent droit au cachot local) ou en leur évoquant des écrits sacrés et secrets que certains seraient susceptibles de monnayer. 
Écritures nocturnes, régénération et circulation des écrits chez les Mayas cruzo'ob

Valentina Vapnarsky et Hilario Chi Canul

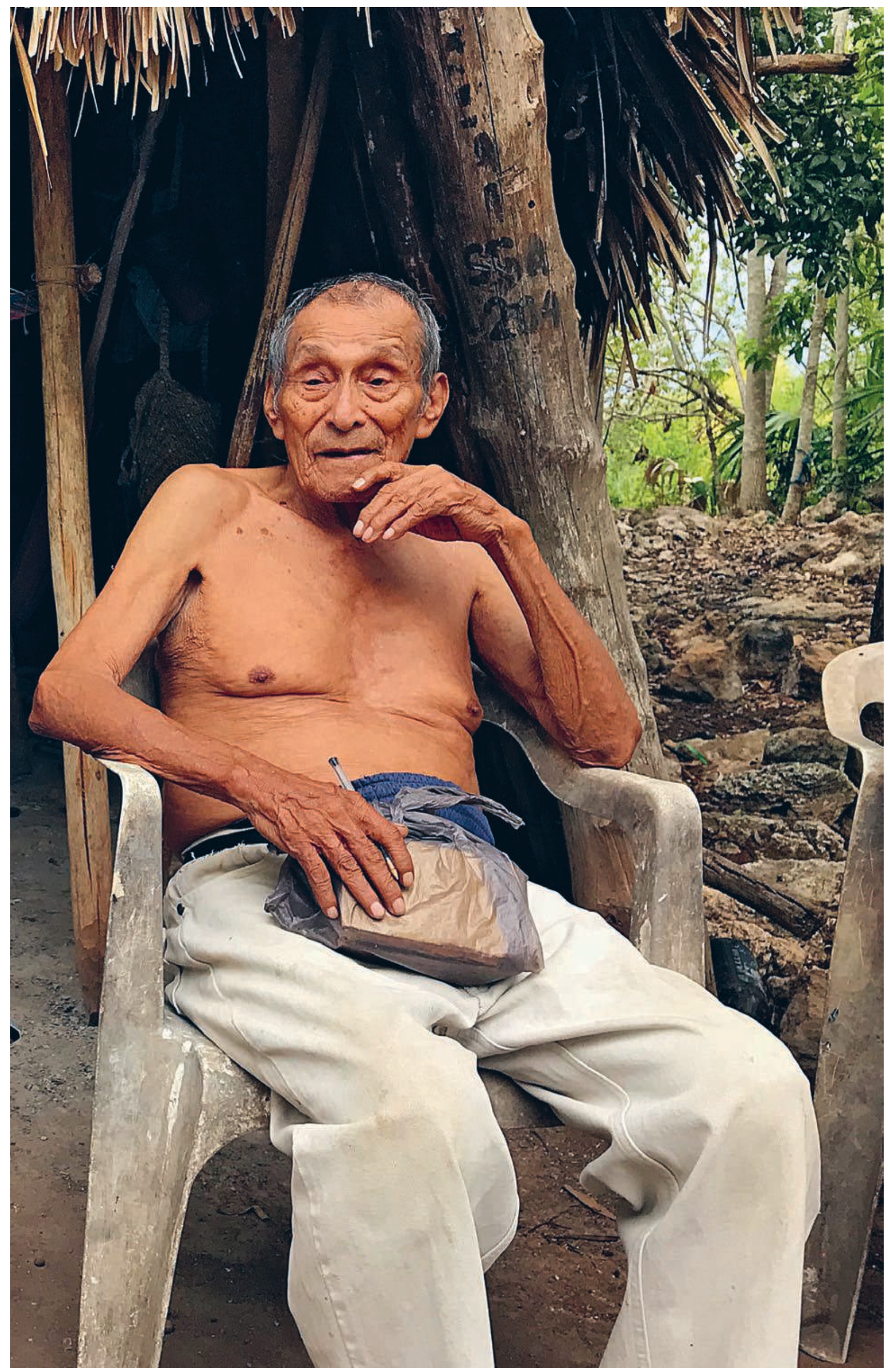

Aniceto May Tun devant chez lui, tenant quelques-uns de ses cahiers, x Pichil (Quintana Roo, Mexique), 2019. Photo: Valentina Vapnarsky. 
9. Termes toujours utilisés comme emprunts de l'espagnol en maya.

10. Le bâtiment est aujourd'hui connu comme Akab Dzib (suivant une orthographe maya courante au $x x^{e}$ siècle) et répertorie comme la structure 4D1 du site, datant du Ix siècle.

11. Le terme maya yum désigne basiquement «père, maître, possesseur».
«Aha, répondis-je au message de mon invité. Pourquoi pas. Je veux bien voir ces écrits si ça peut t'être utile. Peut-être pourrais-je t'aider.»

Les quelques paroles d'Hilario avaient suffi à aiǵuiser ma curiosité.

La nuit était déjà tombée depuis longtemps. Nous n'avions cessé de discuter depuis son arrivée à Paris, quelques jours auparavant. Hilario était allongé dans le hamac de mon bureau quand nous en vînmes à évoquer les textes anciens qu'il avait rapportés. Il commença par me narrer avec les mêmes détails que ceux rappelés plus haut comment il avait reçu ces écrits et le cauchemar qu'il avait fait. Je devais être prévenue. Nous convînmes que mieux vaudrait étudier les cahiers le lendemain matin.

Le jour levé, Hilario revint avec une petite boîte en carton entre les mains. Nous nous assîmes tous deux à mon bureau, il déposa la boîte sur la table puis l'ouvrit délicatement. J'aperçus alors trois petits carnets d'une dizaine de centimètres de large et une quinzaine de haut. Il m'en tendit délicatement un, que je commençai à feuilleter. En parcourant lentement les pages, je vérifiais les éléments de la description qu'il m'en avait faite: les textes en maya, le mélange des genres, la variété des écritures et des encres, les signnes qui illuminaient de nombreuses pages et que je percevais plutôt comme des étoiles minutieusement rayonnées ou parsemées de pointillés, des séries de traits fins se déroulant en lignes serpentines, telles les jeunes tiges d'une plante grimpante cherchant leur chemin, ou des enluminures de lettres et de mots. Il y avait aussi les comptes de chicle, et la fameuse mention de Noj Kaaj Sáanta Kruus x Báalam Naj K'anpok'olche' Kaaj, nom du villaǵe sacré, qui apparaissait à plusieurs reprises, mais toujours plutôt griffonnée, dans quelques recoins des carnets. Je découvris aussi d'autres genres textuels et de nouvelles ressemblances: de nombreuses pages des carnets étaient couvertes de prières, certaines en maya mais aussi en latin, tracées d'une belle plume très habile ou, à d'autres endroits, au stylo à bille d'une main plus maladroite. La plupart de ces prières étaient ajourées par les formes dessinées. Mais il y avait également de longues pages entièrement tapissées de récits en maya, presque sans respiration. Alternant nos voix, nous nous mîmes à en déchiffrer certains fraǵments, pour y découvrir des histoires de chasseur, de cerf, de soldat et puis d'homme transformé en cheval, de bateau voguant en mer; apparaissait aussi Ponso Pilatu, un ermite, de jeunes vierges, Lucilo, le Sancto Papa ou encore Romae. Nous ne comprenions tout cela que très sommairement. Les tournures étaient à la fois familières et inusitées; j'y reconnaissais autant des caractéristiques linguistiques du maya colonial que des marques d'oralité encore courantes dans les récits actuels, telle la façon dont les dialogues se voyaient mis en scène en direct, par un jeu de citatifs spécifiques. Si Hilario était fasciné par les enluminures, pour ma part, j'étais tout autant subjuguée d'entendre ces carnets me raconter dans un style si vivant des histoires sorties droit de l'époque coloniale. Et il y avait plus encore, comme ces récits de la Passion, chemins de Croix déroulés en maya, puis plus sommairement en espagnol. Et on trouvait aussi, imbriqués ou superposés entre ces textes, une quantité de petits blocs d'écritures, le plus souvent avec des dates, des noms de personnes et de lieux. Il semblait s'agiir dans maints cas d'actes ou de rappels de décès, notés scrupuleusement là où le scribe avait pu trouver de la place. Sur l'un des carnets étaient inscrites des dates s'étalant, au fil des paǵes et sans ordre chronologique apparent, entre 1904 et 1970. Enfin, il y avait ces sortes de gribouillage au crayon à papier, des imitations d'écriture esquissées par une main analphabète, peut-être celle d'un enfant.

\section{ÉCRITURES NOCTURNES}

Hilario avait fait ce rêve terrifiant. Ces carnets, lui avait-on rapporté, étaient des áak'ab ts'íb, des «écritures nocturnes», un terme qu'on employait rarement. De ces écritures, on disait qu'elles étaient invisibles de jour et saillantes de nuit. Étaient-elles des écritures de sorcellerie? Les sorciers, racontait-on, apprennent par les livres; il y avait les livres de magia blanca et ceux de magia negra ${ }^{9}$. Ces livres se lisaient plus qu'ils ne s'écrivaient, même si l'on racontait que certains sorciers avaient été surpris avec des cahiers emplis de signes et de dessins "de mauvaises choses », alors qu'ils n'avaient jamais appris à écrire. Chez les Cruzo'ob, Paul Sullivan semblait indiquer que le terme áak'ab ts'iib, pouvait aussi être employé pour désigner les prophéties, même si c'était un usage peu fréquent (Sullivan 1989: 4). Quelques décennies plus tôt, des Mayas qui faisaient découvrir les ruines de Chichen Itzá envahies par la forêt à John L. Stephens indiquèrent par ce nom - que l'explorateur nota Akatzeeb et qui fut plus tard rendu par «écritures mystérieuses »- un bâtiment où se trouvaient des écritures ǵlyphiques anciennes. Leur signification et leur perception étaient obscures: outre qu'elles étaient indéchiffrables, ce savoir s'étant perdu depuis plusieurs siècles, elles se trouvaient cachées dans une pièce où régnait la pénombre ${ }^{10}$ (Sullivan 1989: 9; Stephens 1848, vol. II: 204 ff.). D'autres sens sont succinctement rapportés dans les dictionnaires coloniaux : ceux d' «écritures chiffrées», mais également d' «abréviations». La seule certitude qui s'en dégage est qu'áak'ab ts'îb désignait et désigne encore une chose occulte, requérant une clef d'interprétation pour être comprise - tels les ǵlyphes, les abréviations et les prophéties - ou ne se révélant que par transformation - tel le passage du jour à la nuit.

Les jrwaay, qu'on disait être les maîtres ou propriétaires ${ }^{11}$ des carnets, se transforment eux aussi avec la nuit. Selon leur nature, ils peuvent se changer en chien, en bouc, en chat, en cochon, en dindon, certains mêmes deviennent des jaǵuars, de grands oiseaux noirs ou des nattes volantes... Chaque jwaay (ou xwaay s'il s'agiit d'une femme) a un double privilégié. Sous cette forme, il ou elle pénètre d'autres maisons pour tourmenter leurs habitants. Le terme pour waay est issu de la même racine lexicale que le mot wayak' qui se réfère aux rêves très signifiants du type de celui qu'avait fait Hilario. Auparavant, 
waay renvoyait plus largement au nom du double, généralement animal, que chaque individu était susceptible de posséder et avec lequel il partageait une coessence (connu dans la littérature comme nahual ou tonal). À l'époque préhispanique, au moins pour l'élite qui produisit les textes en écriture glyphique, le waay était conçu comme une entité émergeant de l'inframonde et de la nuit, pour transmettre certains savoirs experts et ésotériques, tels que les connaissances calendaires ou arithmétiques, mais aussi la maîtrise de l'écriture. Ces savoirs impliquaient le waay serpentvision, ou sa manifestation décharnée plus directement associée à l'inframonde, le mille-pattes. On trouve ainsi nombre de représentations iconographiques où le serpent, et plus souvent le mille-pattes, entoure ou prolonge le corps d'un scribe, sous son apparence humaine ou simiesque ${ }^{12}$. En outre, le glyphe de la nuit est souvent associé à ces représentations, et plus largement à des scènes d'écritures (Becquey 2020; Ciura 2019; Taube 2003).

Avec la diabolisation des croyances locales par les missionnaires, waay a pris au fil des siècles une connotation essentiellement négative. Aujourd'hui, les waay effraient, ils font du mal et il n'est pas rare qu'ils soient finalement assassinés. Il y a des familles de waay. Les waay n'ont pas besoin de livres pour se transformer et les livres de magia ne sont pas nécessaires non plus pour pul-yah («jeter des sorts»). Ils peuvent toutefois aider, et certains sorciers savent y perfectionner leur savoir.

La nuit suivant sa première manipulation des carnets, un chien noir menaçant s'était approché d'Hilario et l'avait rempli d'effroi. Qu'il s'agisse d'un rêve n'ôtait rien à la réalité de cette visite. Hilario avait ensuite appris que le jwaay qui possédait les carnets se transformait en grand chien noir. Et plus tard encore, nous découvrîmes que la première épouse dudit sorcier avait été tuée, alors qu'elle agissait également sous la forme d'un chien noir. Ainsi transformée, racontait-on, elle s'immisçait nuitamment chez un jeune homme qui, chaque jour, s'éveillait entièrement dénudé, le pénis tuméfié (Sullivan 1989: 201). «Eh bien, ce devait donc vraiment être le pixan [ «l'esprit»] du grand homme qui m'avait rendu visite cette nuit-là », en avait conclu Hilario.

\section{LE SORCIER AUX PARABOLES OU LA FIN DU MONDE}

Ce jwaay était un sorcier fort réputé et craint. Au-delà des informations fournies oralement, nous avions retrouvé trace de son nom, par le biais de deux petits feuillets de convocation à des assemblées restés dans les carnets. Il s'agissait de Santiago Itzá. Don San, comme on l'appelait, était un personnage connu dans la région. J'avais d'ailleurs eu le privilège de le rencontrer, plus de vingt ans auparavant, par le biais d'un homme maya de Carrillo, politicien aguerri, qui officiait comme truchement entre les Mayas et le gouvernement ${ }^{13}$. Il négociait des affaires avec le vieil homme. Il me l'avait présenté comme une personne de grand savoir, qui maîtrisait l'art des «paraboles », et m'avait un jour conduite chez lui, dans son village situé au nord de la réǵion cruzo'ob. Après une lonǵue conversation entre les deux hommes sur des questions politiques, sa seconde épouse nous avait offert du chimole $^{14}$ de la fête de la veille. Don San était plein d'humour et très loquace. Il nous avait montré sa large collection de photos. Une partie d'entre elles, tirées en assez grand format, étaient «historiques», avais-je noté dans mon carnet de terrain : j'en reconnus immédiatement plusieurs qui avaient été reproduites dans le livre de Villa Rojas (1987 [1945]), dont le fameux portrait du capitaine maya Cituk qui ornait la couverture de l'édition mexicaine, pris par Francés Rhodes Morley, l'épouse de l'archéologue. À l'arrière-plan de l'une des photos, Don San me signala son père. Ce dernier, Don Apolinario Itzá, avait été «scribe» (jts’íb, eskribaano) et «secrétaire» (sekreetaryóo), m'expliqua-t-il. Il me montra également un sceau de cire rouge gravé d'une inscription qu'on percevait mal et que je ne compris pas; il l'avait trouvé sur une lettre appartenant à son père et l'en avait séparé, me précisa-t-il ${ }^{15}$.

Don San repartit avec nous à Carrillo où il devait assister à une réunion. Sur le chemin du retour, nous aperçûmes des maisons détruites par le cyclone qui avait violemment balayé la région quelques semaines auparavant. Un cyclone sec, sans pluie, commenta-t-il, bien différent de ceux déjà connus. Savais-je bien comment l'on disait cyclone en maya? «Chak ik'al.» G'était un présage important: «bíin éelek yóok'ol $k a b$. ( «La terre brûlera. ») L'énoncé, aux tournures requises pour la prédiction, faisait partie d'un répertoire prophétique communément partagé (Sullivan 1984; Vapnarsky 1996). On savait que l'humanité actuelle aurait un terme, comme les précédentes qui avaient décliné et disparu - du moins provisoirement. Mais le temps et la forme que prendrait cette fin restaient incertains. Don San enchaîna avec une histoire, celle d'animaux sylvestres assoiffés, fuyant le feu:

«Un à un, ils accouraient vers toi et te demandaient: "Je'lena'! Tak ajaantkeni'?" ["-Me voici! Veux-tu me manger?"] Et tu répondais: "Máa'! " ["Non! ”] Chaque jour, un nouvel animal venait ainsi t'interpeller. D'abord le xk'ankabtsutsuy, un petit oiseau couleur de terre noire qu'il était rare d'apercevoir. Il s'approchait car il n'avait plus où puiser de l'eau. Puis c'était le singe; le lendemain, le pécari à lèvres blanches: "Kínseni"! Kínsen ajanten! Janteni”!" ["Tue-moi ! Tue-moi pour me manger! Mange-moi !"] disait-il. Mais toi, tu n'avais aucune envie de les manger; car tu sentais la fin s'approcher. Le lendemain encore, le pécari à collier venait à toi, un nouveau jour se levait et c'était l'aǵouti paca, puis l'agouti rouge, et le surlendemain, le dindon ocellé, et aussi le grand hocco, puis la perdrix, et même l'ortalide chacamel; puis un autre jour naissait et le coati arrivait, puis l'écureuil, le lapin, et même le cochon d'Inde, ils venaient tous à toi les uns à la suite des autres, jour après jour, t'implorer: "Je'lena'! Janteni'! " ["Me voici! Mangemoi!"] "Wa ma' ajanteni', 'aalak'teni', ki tech." ["Si tu ne me manges pas, apprivoise-moi au moins"], te priaient-ils. Mais toi tu ne les mangeais pas. Tu les vois tous défiler en courant. Ils parcourent la terre
12. Le mille-pattes émerge souvent du dos du scribe, telle une longue queue en volutes (vase K1523), suivant sa courbe (Yaxchilan, linteau 48); un autre bas-relief révèle la gueule ouverte du mille-pattes d'où émerge une main de scribe manipulant un stylet (Tikal, sépulture 116) (Taube 2003: 414; Ciura 2019: 69, 71, 72).

13. Les rencontres relatées à la suite eurent lieu entre le 13 et le 20 décembre 1995. Carnets de terrain de l'auteure, $13^{\prime}$ (p. 173-180) et 14' (p.131-151)

14. Plat constitutif des offrandes réalisées lors des fêtes patronales.

15. Le sceau représentait un glyphe maya. Il avait été donné par Morley au jeune San lors de leur dernière entrevue. San l'avait interprété, lui, comme un emblème des États-Unis et un signe de reconnaissance en vue d'alliances futures (Sullivan 1989: 184). Il n'était pas étonnant qu'il l'eut montré à l'étrangère que j'étais, et qui manqua là une occasion importante. 


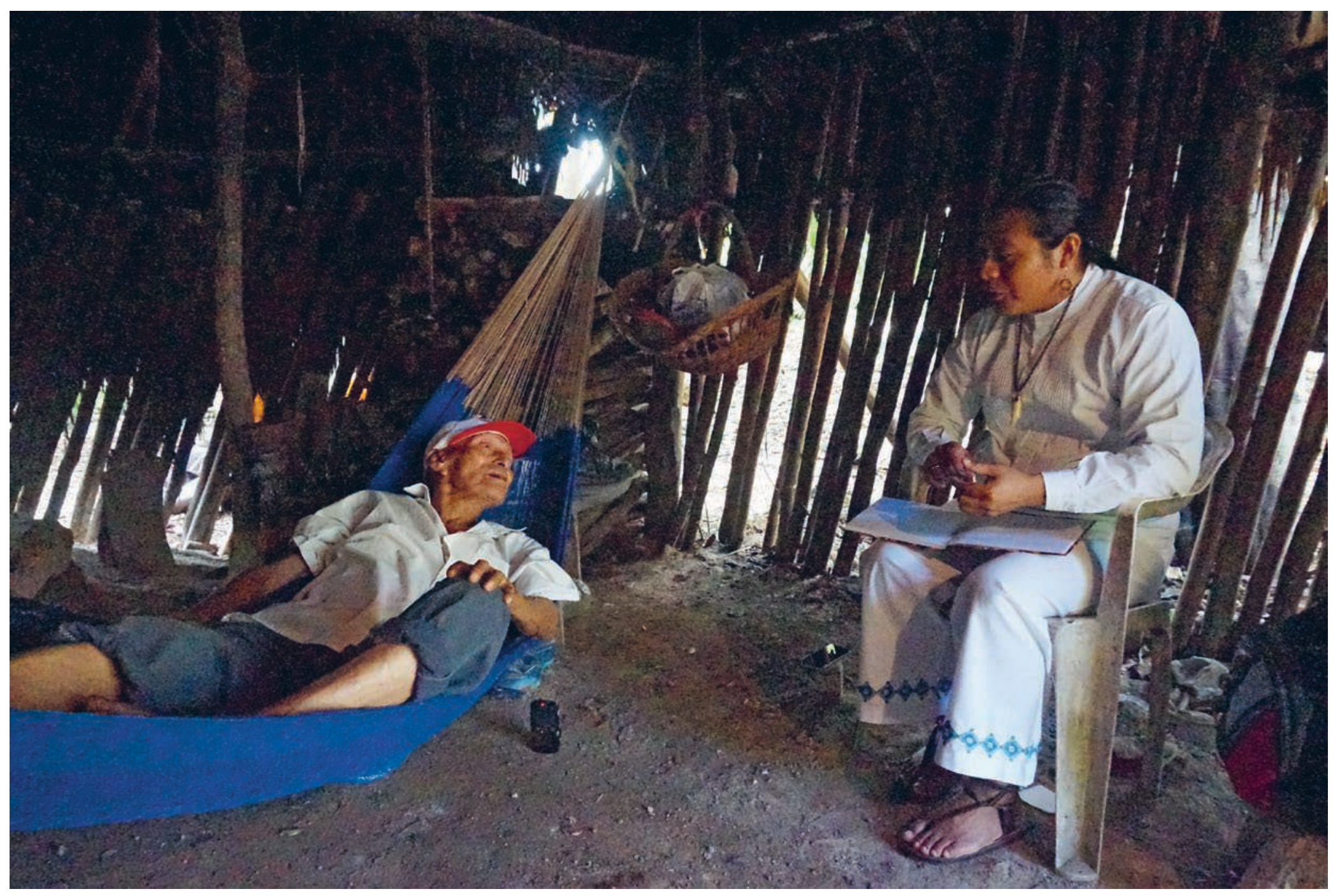

Aniceto May Tun et Hilario Chi Canul, x Pichil (Quintana Roo, Mexique), 2019. Photo: Valentina Vapnarsky. 
entière pour aller vers toi. Et quand ils seront tous passés, conclut Don San: "Xuulul ken ubeet yóok'ol $k a b$." ["Ce sera la fin du monde."]»

Quelques années auparavant, Don San s'était entretenu avec l'anthropologue Paul Sullivan sur un récit de fin du monde un peu différent (Sullivan 1989: 205 ff.). Enfin, s'aǵissait-il d'un récit? Et était-il si différent? Don San possédait plusieurs manuscrits et documents qu'il disait avoir hérités de son père. Il les montrait parfois aux étrangers qui venaient lui rendre visite et on l'accusait de les leur vendre. Celui qu'il prisait le plus, dit-il ce jour-là à Paul Sullivan, était une «Histoire de Dieu». Pourtant, il ne pouvait pas la lire. En effet, bien que son père ait été le scribe le plus connu de la réǵion, Don San n'avait jamais appris à lire. Cela ne l'avait pas empêché de connaître le texte par cœur, à force d'écouter feu son père le lire à voix haute. Était-ce pour raviver ce souvenir? Retrouver le plaisir du texte lu? Pour tester le nouveau venu? En témoignage de confiance? Ou encore en gage diplomatique?... Le fait est que Don San demanda à Paul Sullivan de bien vouloir lui lire le texte à voix haute à son tour ${ }^{16}$ :

1. Ceci est le premier jour où prendra place le signe, la mer s'élèvera, jusqu'aux grandes vagues, jusqu'aux collines les plus hautes, elle ne s'écartera pas des lieux de rame, elle s'élèvera tel un mur.

2. Ceci est le deuxième jour, il y aura un signe en ce second jour, elle s'abaissera aux lieux de grande profondeur, elle s'abaissera profondément, l'on ne verra pas où elle va, quand elle s'enfoncera sur la plage.

3. Ceci est le troisième jour, il y aura des signes afin que vous les voyiez, elle arrivera là de nouveau comme vient le jour/soleil.

4. Voici le quatrième jour, il y aura ce signne, voici toutes les créatures de la mer, et aussi les poissons, ils se réuniront sur la surface de l'eau en criant de douleur, en se lamentant sur la surface de l'eau, car ils auront peur de la venue du jour final.

5. Voici le cinquième jour, il y aura ce signe, les oiseaux se réuniront tous avec les autres animaux, ils se réuniront tous ensemble, ils seront en train de pleurer aussi, car leur mort approche au jour final.

6. Voici le sixième jour, il y aura ce signe, le feu sortira des airs, à l'ouest pendant que plonge le soleil, et, pendant que sort le soleil, à l'est.

7. Voici le septième jour, il y aura ce signe, il y aura de nombreuses comètes, des éclairs de tonnerre et des étoiles tombant du ciel l'on verra.

8. Voici le huitième jour, il y aura ce signe, il y aura un cyclone, voici les animaux, tous, aucun d'eux ne tiendra debout, aucun d'eux ne tiendra assis non plus. Ils tomberont tous sur la face de la terre, tous. [...]

\section{LES CHILAM BALAM OU L'ENGHEVETTREMENT DES COPIES}

Apolinario Itzá, connu sous le nom de Yum Pol, avait été «scribe» du centre cérémoniel de xCacal dans les années 1930. Il était aussi «secrétaire» de la croix prodiǵieuse, la Santísima, qu'avaient «capturée» en 1929 les hommes de son groupe au village sacré de Chan Kaaj Saanta Krus x Báalam Naj K’anpok'olche Kaaj. Ce vol avait été motivé par des accusations de trahison à l'égard du général maya qui gouvernait depuis ce centre, et il conduisit à la fondation du centre cérémoniel de xCacal autour de la croix. C'était l'époque où Villa Rojas, assistant de Robert Redfield, avait vécu et mené sa fameuse ethnographie à Tusik', un village proche de xCacal, lui-même situé non loin de Señor où résidait Yum Pol 17. Sylvanus Morley, l'archéologue, était installé à Chichen Itzá depuis 1924, et avait travaillé auparavant dans d'autres sites mayas de la région. Yum Pol avait joué un rôle essentiel dans les intenses négociations entre les leaders mayas et l'archéologue, considéré par les Mayas comme un émissaire politique des États-Unis. De fait, Morley avait été un excellent aǵent de la CIA durant toute sa carrière (Sullivan 1989; Harris et Sadler 2003). Comme le résume Paul Sullivan, «en Apolinario Itzá, s'entrecroisaient les canaux de communication avec les étrangers et avec le divin. Même si Vrai Dieu avait cessé d'interpeller les Mayas de façon audible et publique, les officiers [mayas] continuaient à recevoir des lettres qu'Il dictait à Itzá, supposément dans des états extraordinaires d'inspiration et de possession divines. Et c'était Itzá qui lisait les “commandements divins" aux Mayas au centre cérémoniel de xCacal Guardia» (Sullivan 1989: 49-50, nous traduisons).

Comme très souvent, des tensions étaient nées entre les autorités du centre cérémoniel. Yum Pol avait été accusé de profiter de sa relation avec Morley et Villa Rojas, et même d'être responsable de l'échec des négociations avec les États-Unis. Il avait été aussi accusé de sorcellerie. On disait qu'il avait tué l'épouse de l'un des officiers mayas et avait mangé son âme. Un matin de 1943, en quittant la petite maison qu'il occupait près de son champ, il avait reçu des coups de fusil auxquels il succomba aussitôt. Morley mourrait lui aussi peu d'années après, en 1948, à Santa Fe, New Mexico. Quand les Mayas de xCacal avaient appris cette nouvelle, une rumeur avait filé à voix basse: c'était l'Almaj t'aan, l'écrit sacré où s'exprimait Juan de Cruz et dont Yum Pol avait été le principal lecteur, qui avait ôté la vie de l'archéologue (Sullivan 1989: 180).

Une dizaine d'années avant, Yum Pol avait montré à Villa Rojas un petit carnet manuscrit qu'il conservait. Celui-ci comprenait deux couleurs d'encre et des écritures différentes. Une première section se concluait par la signature d'Anastasio Caamal et la mention de la date de 1887 . Les paǵes suivantes avaient été écrites par son fils aîné, Andrés, qui lui avait succédé comme scribe. À son décès, en 1906, la charge serait passée à son frère cadet José Santos, qui était décédé en 1916. Peu avant, José Santos avait divisé les manuscrits entre son fils Marcelino et Apolinario Itzá. Le premier était plutôt versé dans
16. Nous donnons ici un extrait du «Texte des quinze signes» (pour la version intégrale, voir: Sullivan 1984; Grupo Dzíbil 1996; pour d'autres versions, voir: Liljefors Persson 2000; Christensen 2016: 168-171; Whalen 2003: 62-69). Par manque de place, la version maya ne peut être reproduite. Notre traduction se fonde sur la reproduction de l'original dans l'édition de Sullivan (1984: 268-287), elle-même inspirée d'une version inédite d'A. Barrera Vásquez, et d'une comparaison avec le manuscrit de Teabo (Christensen 2016).

17. Après une première incursion en 1932, il y séjourna plus longuement entre 1935 et 1937. 
18. Les plaques photographiques ont rejoint la Carnegie Institution of Washington Collection (fonds Ralph Roys) des Archive (fu Peabody Museum of Archaeology and Ethnology, Harvard University, cité par Sullivan (1984: 265-276; 1991 : 221 et 265, n.4). Selon le Grupo Dzíbil (1996), une copie photographique aurait été faite par Morley, à la demande de la Bibliothèque Newberry de Chicago et aurait rejoint le fonds Morley à I'université de New Mexico.

19. Voir notamment: Bricke et Miram (2002); Caso Barrera 2019 [2011]; Christensen 2016.

20. On notera que le manuscrit aux mains d'Apolinario Itzá setrouvaiten faità Senc se trouvaiten fait où il résidait, et qu'il était lié au centre cérémonial de $x$ Caca dont ce village et le scribe eux-mêmes dépendaient. La désignation de Tuzik renvoie donc au lieu où Villa Rojas vit le texte pour la première fois lors d'une des visites d'Apolinario Itza'.

21. Villa Rojas à Redfield, 26 mai 1936, 4décembre 1936, Box5, Folder7, Robert Redfield Papers, cité par Sullivan 2021: n. 18.

22. Le travail sur ce carnet a été réalisé à trois, en collaboration avec Julien Machault; une publication est en préparation. les arts chamaniques des jmeen, on ne sait pas ce qu'il fit des écrits. Le second deviendrait le scribe principal de xCacal, Yum Pol (Villa Rojas, 1987 [1945] : 459;

Sullivan 2021). Le petit carnet que Yum Pol montra à l'ethnologue en affaires avec les Américains contenait un Almaj t'aan de Juan de la Cruz. Si les évènements qui y étaient consignés se rapportaient à des faits de guerre vieux de plus d'un demi-siècle, Yum Pol répétait néanmoins annuellement la lecture de ce texte devenu écrit fondateur devant un parterre de fidèles, aǵenouillés et tête basse, lors de la grande messe solennelle qui concluait la fête patronale. Lors de l'une de leurs rencontres, à Tusik', Yum Pol avait aussi remis à Villa Rojas et à Morley un manuscrit qui s'abîmait pour qu'ils le lui rénovent (Sullivan 1989 et 2021). Le manuscrit avait bien été rendu à Yum Pol, mais dans le même temps, une copie photographique en avait été faite, qui ensuite avait circulé entre bibliothèques étatsuniennes et mayanistes érudits, tels qu'Alfredo Barrera Vásquez ${ }^{18}$. Puis, d'autres copies photoǵraphiques légèrement différentes du manuscrit étaient semblet-il aussi apparues (Grupo Dzíbil 1996). De l'oriǵinal, on rapportait qu'il s'agissait d'un livre magnifique, illustré de dessins de couleur et relié de peau de cerf. On ne savait pas où il se trouvait.

Le document avait été baptisé «Chilam Balam de Tuzik». Ce nom, sans doute attribué par Villa Rojas, l'insérait dans la prestigieuse série de manuscrits connus sous l'appellation «livres du Chilam Balam». Les plus réputés de ces manuscrits sont les Chilam Balam de Chumayel, de Mani, de Tizimin ou encore de Kaua, du nom des lieux où ils furent trouvés, mais une bonne dizaine ont été répertoriés. Ces écrits furent rédigés en alphabet latin, mais en langue maya, par et pour les Mayas, et conservés avec la plus grande révérence. Palimpsestes au contenu éminemment hybride, la plupart incluent des sections datées de différentes époques, pouvant remonter jusqu'au $\mathrm{XVI}^{\mathrm{e}}$ siècle. Outre des textes historiques et prophétiques, énoncés dans un langagóe ésotérique mettant en œuvre une intrication complexe de temporalités, ils révèlent l'absorption et l'intégration, dès les premiers temps de la conquête, des dits, des écrits et des idéologies transmises par les missionnaires et autres colons. Ils incluent ainsi, selon les versions, des copies ou insertions d'almanachs et d'horoscopes issus de Repertorios de los tiempos, de traités d'astronomie et de sciences naturelles, de textes bibliques et théologiques, des quinze signes du Jugement dernier, ou encore de romans médiévaux ${ }^{19}$. Comme le relève Sullivan, lors du séjour de Villa Rojas à Tusik', l'édition de Ralph Roys du manuscrit de Chumayel venait d'être publiée. Villa Rojas rapporte qu'Apolinario Itzá, Yum Pol, venait souvent lui rendre visite à Tusik', principalement pour écouter la lecture du texte maya du Chumayel. L'ethnologue lui avait même montré que le manuscrit qu'il possédait - celui dit de Tuzik ${ }^{20}$ - «était une version d'une partie de ce Chilam Balam [de Chumayel] » (Villa Rojas 1987 [1945] : 217), relation qu'il commenta aussi à son mentor Robert Redfield (Sullivan 2021: n. 18) ${ }^{21}$. Ces parties contenaient un ensemble d'éniǵmes qui figuraient sous la section «L'interrogatoire aux chefs» dans l'édition de Roys, devenues depuis plus connues sous le nom de «Langage de Zuyua » (Barrera Vásquez et Rendón 1978 [1948]; Ligorred Perramon 2000; Villa Rojas 1984). Ces pages relateraient les épreuves verbales de déchiffrement d'éniǵmes au lanǵage hautement métaphorique, auxquelles les chefs de lignage devaient se soumettre. Il y était fait référence à des dates des cycles calendaires mayas, mais le texte mentionnait également que leurs auteurs, indexés à la première personne du pluriel, avaient rédigé ces éniǵmes en l'an 1628.

Lorsque Don San donna à lire à Paul Sullivan, dans les années 1980, le petit carnet aux feuillets usés, sommairement cousus à la main, recouvert d'écriture sur une quinzaine de pages, l'anthropologue reconnut, rapporte-t-il, le Chilam Balam de Tuzik dont le Peabody Museum conservait des copies photographiques. De fait, le «Texte des quinze signnes de la fin du monde» que le vieil homme lui avait demandé de lire se trouvait aussi, à l'identique, dans la copie. Il supposa que la couverture de peau de cerf avait dû s'en détacher. Il en conclut que le manuscrit, désormais célèbre et recherché, était passé aux mains de Don San, qui le conservait aussi soigneusement que l'avait fait son père (Sullivan 2021).

Nous avions beau lire et relire les pages des carnets, désormais numérisées, qu'Hilario avait été chargé d'étudier, celles-ci ne comprenaient pas les signes de la fin du monde. Il n'y avait pas trace non plus du Langage de Zuyua. En revanche, nous y découvrîmes un ensemble de récits identiques à d'autres présents dans la reproduction du Chilam Balam de Tuzik qui avait jusqu'alors moins suscité l'attention. Il s'agissait des récits intitulés Julian upec tzilah Ceh («Julian l'annonce du chasseur [ou du cerf]) », Ermitaño («L'ermite») ou encore Ahau Pilato («Seigneur Pilate»), écrits dans un maya de facture ancienne, tout en incorporant de nombreux emprunts lexicaux à l'espagnol. Ces récits furent vraisemblablement inspirés d'histoires de saints et martyrs de la Légende dorée de Jacques de Voraǵine, ouvrage qui connut un vif succès au Moyen Âge tardif et dont la diffusion s'accrut encore avec l'imprimerie et l'entreprise coloniale d'évangélisation (Liljefors Persson 2000 : 251). Par ailleurs, si les récits étaient littéralement identiques à ceux du Chilam Balam de Tuzik, une comparaison détaillée du contenu, des calligraphies, de l'agencement et de l'espacement des mots nous révéla que nous avions entre les mains une copie différente du même texte 22 Certains traits conduisent à penser qu'elle lui est postérieure. Mais avait-elle été directement copiée de celle montrée à Villa Rojas? Une autre copie encore avait-elle servi de modèle au document que nous analysions? Ces questions restent ouvertes. Et Don San avait-il eu entre les mains les deux copies ou d'autres versions encore simultanément? Cela était fort possible. Plusieurs des carnets reçus et qui provenaient du vieil homme étaient eux-mêmes unis par des liens textuels ou matériels et des jeux de copie.

Le carnet des récits ne comprenait ni les signes du Jugement dernier ni les paroles de Zuyua. En revanche, entre la couverture et la première page avaient été ǵlissés d'autres feuillets. Écrits d'une belle plume et à l'encre, ils avaient été consolidés bien plus 
récemment par une reliure de fortune, composée sur la fine tranche à l'aide d'un bout de carton arraché à une boîte de médicament Pfizer ${ }^{23}$. Ces pages étaient couvertes de prières en latin.

\section{LES PRIÈRES ET LA RÉNOVATION DES ÉCRITS}

San, fils aîné de Yum Pol, avait bien failli être assassiné aux côtés de son père. Sa mort avait été également planifiée, disait-on, mais certains hommes, apitoyés par son jeune âge, l'avaient sauvé (Sullivan 1989; 2021). Don San n'avait appris ni à lire ni à écrire (ibid). Dans les termes mayas, on dirait qu'il n'avait pas reçu le don de l'écriture, ma'uti'ali' («ce n'était pas à lui »), c'est-à-dire pas pour lui. En revanche, il était devenu jmeen, et même un jmeen très recherché. Les jmeen n'utilisent pas l'écriture. Leur apprentissage est informel et se développe loin des cahiers de prières ou d'autres artefacts scripturaux. Aǵissant souvent comme assistants de jmeen expérimentés durant plusieurs années avant d'exercer eux-mêmes ${ }^{24}$, ils acquiérent des savoirs pratiques et oratoires considérés comme issus de l'enseiǵnement reçu des esprits gardiens du monde sylvestre, lors d'expériences oniriques ou extatiques dans la forêt. Le jmeen, dit-on, «lit» (xok) les reflets des cristaux divinatoires sáastun, la flamme et la lueur des bougies, et même le visage de ceux qui viennent le consulter et desquels il attend peu d'explications. Il voit et interprète. En revanche, un jmeen dont on découvre «qu'il apprend avec des livres » est considéré comme un charlatan.

L'apprentissage non scriptural des compétences ésotériques des jmeen se distingue nettement de la relation à l'écrit qui caractérise l'instruction et la pratique d'autres hommes rituels et orants remarquables: les prieurs reesa (emprunt dérivé de rezar, «prier» en espagnol). Spécialisés dans la liturgiie dédiée aux Santisímas et aux saints, ces derniers apprennent avec des cahiers, en copiant, recopiant ou transcrivant des prières. De ces prières, en maya mais aussi en latin, les carnets en possession de Don San, jmeen analphabète, étaient remplis. Les deux compétences, celles de reesa et de jmeen ne sont pas exclusives l'une de l'autre et il arrive que des hommes les cumulent. Cela avait été semble-t-il le cas du père de Don San, le secrétaire de la Croix Apolinario Itzá, mais Don San n'avait, au grand dam de ce dernier, suivi que la voix du chamanisme (Villa Rojas 1987 [1945] : 218; Sullivan 2021). Pourtant, il conservait précieusement une collection hétéroclite de textes, la plupart sans doute hérités de son père, certains d'entre eux transmis par une ou peut-être plusieurs générations de scribes antérieurs, telle une "réserve vivante et décentralisée de ce qui constituait pour les Mayas de la région les écrits mayas les plus importants » (Sullivan 2021, nous traduisons). Pour quelles raisons? Après le décès de son père, les autorités et prieurs de xCacal venaient régulièrement consulter et recopier des parties de ce corpus auprès de lui. San avait alors un statut important dans la hiérarchie du centre cérémoniel (Sullivan 2021). Jusqu'au jour où de violents conflits avaient mis fin à cette pratique qu'on pourrait presque qualifier de bibliothécaire. La possession des écrits avait sans doute pris plus de valeur encore à ce moment-là, y compris vis-à-vis des étrangers. Mais l'histoire ne s'arrête sans doute pas là. En tout cas, Don San semble avoir continué à accumuler et réparer des úuchben ts'íb («anciens écrits ») jusqu'à sa disparition.

Une grande partie des écrits présents dans les carnets qui nous avaient été décrits comme áak'ab ts'íb contenaient des prières du genre reesa. Je reconnus le genre de façon générale et plus spécifiquement le Salve et le Suba suba. Avec l'aide de Sara Shroukh, versée dans les oraisons latines, nous identifiâmes le primo nocturno de l'Officium pro defunctis. Je connaissais quelques-unes de ces prières pour y avoir moimême été instruite lors de mon premier long séjour dans la région. Suivant alors l'apprentissage de deux jeunes prieurs, j'avais aussi pu observer l'importance qu'y revêtait la copie de carnets de prières personnels, intégrant des parties sémantiquement obscures pour les orants, qu'ils apprenaient à l'aide de l'écrit. Près de vingt ans plus tard, quand je demandai à ceux qui étaient devenus des prieurs reconnus ce qu'ils avaient fait de leur carnet, chacun me dit à sa façon qu'il l'avait jeté depuis longtemps! Le cahier s'était abîmé et, surtout, depuis qu'ils savaient les prières, il ne leur servait plus. L'un d'eux était toutefois en train d'en réécrire un nouveau. Ce cahier était plus précis que le précédent, m'expliqua-t-il: maintenant qu'il maîtrisait mieux l'art d'énoncer et de chanter les prières, il en avait inventé une nouvelle notation plus exacte. Par exemple, il y répétait les voyelles pour marquer certaines tenues de la voix ou adaptait la séparation des mots sur le papier à son rythme d'oraison. Même si la calligraphie entre ces cahiers récents et les vieux carnets que nous tentions de comprendre était très distincte et qu'il y avait des différences entre les textes des prières, même si mes amis prieurs eurent du mal à déchiffrer cette écriture ancienne et restèrent perplexes devant les formes dessinées qu'ils y découvrirent, et même si pour eux le latin était plutôt du jach maaya «maya authentique», je reconnus dans ces séries plus récentes de cahiers la même pratique de copiage et recomposition que celle des carnets anciens.

\section{*}

«Qu'est-ce que c'est bien écrit! Ce sont des écrits anciens! Vraiment! Mais quelle belle écriture! Celui qui les a écrits savait vraiment! Ce devait être un maestro. Mais où avez-vous pris ces carnets?»

Le vieil homme se mit à lire, sans cacher sa curiosité ni son plaisir. Suivant les lettres, il entonna: «Rekeen keteeknaam donais dominee et' lu perpeeetua lucia eiiis Neneee te adoreemuuus... ». Malgré son grand âge - on disait qu'il avait près de 110 ans -, la lecture lui était étonnamment facile, que ce soit en maya ou en latin. Après l'avoir laissé parcourir plusieurs pages du carnet, nous l'orientâmes sur celle qui portait la mention d'un nom : «Aniceto May Tun », apposé à la façon d'une siǵnature et souliǵné
23. Il s'agit de la boîte d'un flacon de Combantrin, un antiparasitaire courant dont le début de commercialisation date des années 1970.

24. Don San était lui-même parti deux ans suivre un tel apprentissage (Sullivan 2021). 

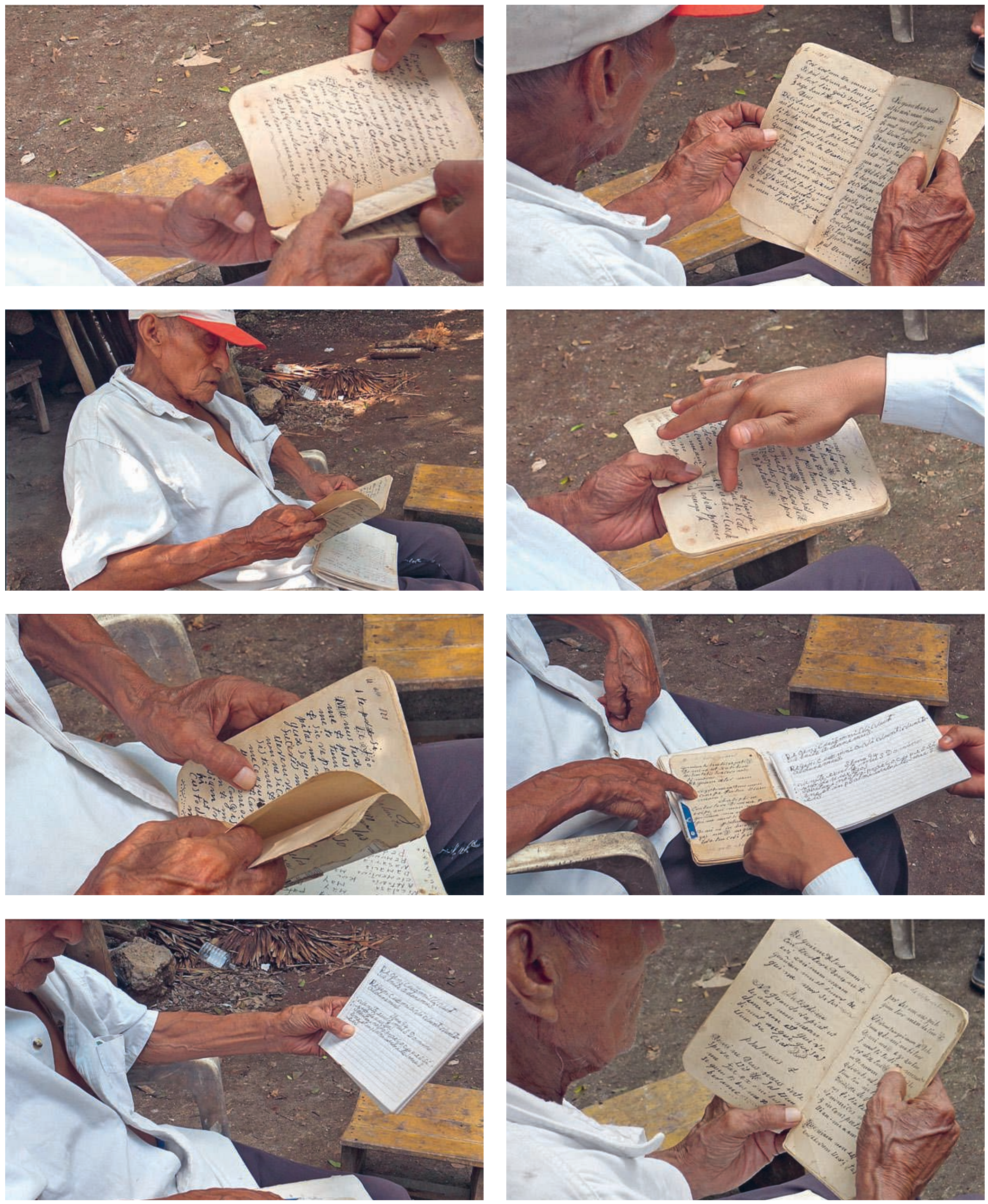

Photogrammes montrant Aniceto May Tun et Hilario Chi Canul observant et comparant des cahiers de prières anciens et récents, x Pichil (Quintana Roo, Mexique), 2019. Vidéo de Valentina Vapnarsky. 
d'une fine liǵne ondulante à franges - ou était-ce un long mille-pattes se déplaçant avec les lettres sur son dos?

«Reǵardez, grand homme... là », Hilario lui siǵnala les lettres de la main. Le vieil homme s'y arrêta et lut à voix haute:

«Aniceto May Tun». Après un court silence, son regard s'illumina:

«Si lela' in ruubrika! Teen! Lelo' teen ts'íibtej! » («Mais ça, c'est ma "rubrique”! C'est moi! C'est moi qui l'ai écrite!»)

Le centenaire avait le sourire d'un jeune enfant. Nous avions retrouvé Don Aniceto un peu par hasard, après avoir vu son nom mentionné dans le catalogue d'une exposition de photos (Barbeau 2017 : 42-45). Il habitait un autre village, xPichil, sur un sentier reculé, passant une grande partie de son temps seul, allongé dans son hamac accroché aux poutres d'une vieille bicoque toute penchée. Sa surprise à la vue de l'un de ses anciens écrits avait été à la mesure de notre ébahissement. Nous avions encore peine à croire que nous avions retrouvé, en vie, l'un des scribes des cahiers parmi les plus anciens de notre collection, que nous pensions d'une époque révolue. Cette visite inaugura une série de plusieurs autres, qui durèrent des jours entiers et durant lesquelles Don Anis ne fut pas avare de paroles. D'une lucidité et d'une clarté remarquables pour son âge, d'une éloquence envoûtante, il nous narra pléthore d'histoires anciennes, du temps où, jeune encore, il s'enthousiasmait d'être autorisé à aller «tuer des Waach» (les soldats mexicains), ainsi que de ses heures passées à apprendre à lire et à écrire «en maya, latin, hébreu et grec». Il avait suivi l'enseignement de son oncle, Dionisio Itzá, qui se trouvait être le frère du fameux scribe Apolinario Itzá. Il avait également été envoyé par son père dans un pensionnat où des érudits mayas enseiǵnaient à un ǵroupe de jeunes Mayas l'art de l'écriture comme celui des paraboles ${ }^{25}$. Aniceto Maya Tun avait ensuite occupé la fonction de secrétaire et scribe de xCacal durant plusieurs années (Sullivan 2021).

Nous le fîmes parler des carnets dont il nous lut de larges extraits. Il nous expliqua comment déchiffrer les diverses formes qui accompaǵnaient ou ornaient les lettres des prières. Ce qu'Hilario avait vu comme des papillons, et moi plutôt comme des étoiles, indiquait ainsi «les moments où le prieur pouvait accepter sa "célébration" », le petit verre d'alcool qui circulait continûment durant les rituels, en d'autres termes les mots après ou avant lesquels il était autorisé à faire une pause dans son flux d'oraisons. Les lignnes continues sous forme de vaguelettes décorées de pointillés indiquaient quant à elles une tenue prolongée et vocalisée de la voix. Les points qui entouraient les mots pouvaient signaler le commencement d'une section mais ils étaient aussi «la force de la prière», ils étaient là pour que «tu aides le chant». Enfin, ce qu'Hilario repérait comme un mille-pattes sous le nom «Aniceto May Tun» singularisait «sa rubrique», sa signature, et manifestait sa présence. Toutes ces marques composaient ainsi une partition qui guidait la mise en voix de la prière et participait de sa force, en aidant les paroles à atteindre ses destinataires, qu'ils soient divins ou défunts. Don Anis nous expliqua aussi les prières en latin, et notamment la composition des différents psaumes de la Vigilia, dont on trouvait plusieurs copies dans les carnets. Il fallait la chanter pour éteindre le feu qui tourmente les défunts et les sauver du purgatoire. On la chantait toujours pour des défunts précisément nommés lors des rituels du souvenir des morts, et c'était peut-être eux dont le nom était parfois inscrit dans les carnets, proposa le vieux prieur, même si lui n'avait pas cette habitude. Il reconnut certains écrits comme étant siens, et fut catégorique sur le fait que le reste avait été écrit par d'autres mains.

Mais où avions-nous pris ces cahiers? La question revenait au fil de nos conversations et appartenait à une longue histoire d'accusations et de suspicions de vol et de vente de vieux écrits sacrés, notamment à des étrangers, dont Don Aniceto avait probablement été lui-même l'un des protagonistes par le passé (Sullivan 2021). Nous n'en savions pas les détails. Notre réponse arriva néanmoins par étapes et sur plusieurs jours, suivant le cheminement lent de la distillation des informations chez les Mayas, ainsi que les méandres du parcours des cahiers eux-mêmes. Nous finîmes par suggérer que, selon les ouï-dire, avant d'être trouvés et récupérés par l'ex-maire, Don San les avait eus entre les mains avant de mourir.

«San Itzá!!! Impossible. Il ne comprenait rien à cela !»

San ne savait rien de ces prières. Don Anis nous conta alors ses faits de sorcier, l'histoire du serpent rapportée plus haut et comment il l'avait lui-même menacé de le tuer sur-le-champ d'un coup de fusil s'il essayait de lui faire du mal. Plus tard, il nous dit toutefois se souvenir qu'il avait bien donné ce cahier à San, du temps où ils étaient amis et que ce dernier s'intéressait aux reesa...

Nous lui demandâmes s'il souhaitait récupérer le sien. Non, puisqu'il le lui avait donné. Et à quoi bon? Pourquoi le voudrait-il? Il en avait de nouveaux. Il nous avait en effet montré un cahier d'un modèle bien plus récent, dont plus d'une centaine de pages étaient couvertes de prières, écrites en diverses encres, mais toutes de sa main. Il nous apprit qu'il était en train d'en copier encore un nouveau: «kintu'umbenkúunsik.» («Je le rénove.») D’ailleurs, le cahier que nous lui apportions était bien vieux et s'abîmait. Le mieux serait que nous le lui laissions pour qu'il nous le recopie, ainsi il nous le «rénoverait». Il faudrait simplement que nous lui apportions un bon cahier vierǵe et de quoi écrire.

Aniceto May Tun n'avait pas que des cahiers dans son antre aux trésors. Dans une maison attenante, en bien meilleur état que celle où il dormait et au milieu de ce qui pouvait sembler un fouillis de vêtements, d'ustensiles et de maigres provisions, se cachait sa bibliothèque: un sac suspendu à une haute poutre, contenant lui-même plusieurs sacs en plastique protégeant les écrits. Une fois, il nous sortit une liasse de feuilles un peu sales, écornées et désordonnées
25. Voir aussi le récit de vie d'Aniceto Maya Tun dans Bracamonte y Sosa et Ek Ek 2014: 59-67. 
qu'un gringo (étranger) lui avait laissée, avec une énorme loupe, pour qu'il les lui déchiffre. Nous comprîmes rapidement qu'il s'agissait de mauvaises photocopies d'une reproduction du Chilam Balam de Chumayel. Don Anis nous avoua qu'il avait un peu de mal avec ce jeu d'úuchbe'en ts'íib («écritures anciennes »). Une autre fois, quand nous l'interrogions plus spécifiquement sur le nom et la fonction des prières manuscrites dans ses anciens carnets, il nous apprit qu'il possédait le livre d'où il en avait copié plusieurs. Il partit à nouveau dans sa maison d'archives et en ressortit cette fois avec un volume imprimé très épais, qui tenait à peine dans sa main. D'un ton assez fier, il nous précisa qu'il l'avait envoyé longtemps avant à Mérida (ville se trouvant à plus de cinq cents kilomètres de son village) pour qu'on lui fasse une nouvelle reliure. Il en ouvrit la couverture. Sur la première page nous lûmes en espaǵnol et en ǵrandes lettres d'imprimerie: Ritual de la oración nocturna («Rituel de la prière nocturne»). Le souffle coupé, nous lui demandâmes d'une même voix:

«Était-ce cela, l'Aak'ab ts'íb [“l'écriture nocturne"]?

- Áak'ab ts'iib?! Ah ma' lelo' yaanam ba'a. Teene', ma' inwoojel le beyo'. Ma' ti teeni'. ["L'écriture nocturne?! Ah non, ça, ce sont des choses différentes. Moi, je ne sais rien de ça. Ce n'est pas pour moi."]»

\section{ÉPILOGUE}

L'analyse de la matière, du contenu et des cheminements des carnets au gré des mains mayas et non mayas, de ceux qui les ont écrits, oralisés ou étudiés - scribes, prieurs, sorciers, politiciens, anthropologues - nous permet de reconstruire des fraǵments de vie des écrits et de leurs manipulateurs, tout en apportant des éléments de réflexion sur l'écriture dans ce contexte culturel et sociohistorique. Écrits de combat, diplomatie et sorcellerie; écriture et transformation; séries et assemblages d'écrits; copies et rénovations; oralisation et scripturalisation sont autant de facettes des conceptions scripturaires mayas que mettent en exerǵue les parcours des carnets étudiés.

Pour les Mayas cruzo'ob, le geste scriptural s'insère dans des actions de fondation, de combat et de diplomatie liées à un contexte de guerre et de recréation sociale. Qu'il s'incarne en des textes fondateurs, des échanges épistolaires ou des recueils de prières, ce geste est toujours associé à une inspiration divine. Il témoigne d'une appropriation fervente de l'écrit comme objet et pratique emblématiques du pouvoir colonial, sous ses versants politiques et religieux. Les Mayas cruzo'ob indigénisèrent de façon particulièrement intense les fonctions sociales et les modes et savoirs scripturaux attachés à l'écriture alphabétique, ainsi que certaines des relations cosmologiques qu'ils sous-tendaient. Le pouvoir de l'écrit se voit aussi instrumentalisé dans les conflits entre factions, lesquels se résolvent communément sous la forme d'accusations de sorcellerie.
La relation entre écrit et sorcellerie s'inscrit plus fondamentalement dans l'opération de transformation révélatrice et son rapport à la nocturnité, que portent tant l'écriture áak'ab ts'iib que le waay, et qui s'instancie sous deux aspects: opacité, car le mode sémiotique est complexe et son message reste invisible pour qui n'en a pas la clef; régénérescence, car la nuit et l'inframonde sont des lieux de transformation et d'émergence génératrice qui permettent l'interprétation. Ces associations sont présentes dès l'époque préhispanique (Becquey 2020). S'y ajoutent alors celle de la matérialisation de l'apprentissage de l'écriture par la figure du waay, puissance émergeant de l'inframonde, et sa représentation sous l'apparence du millepattes. Avec autant de trouble que de suspicion face à une analogie très spéculative, il est saisissant de découvrir à plus de dix siècles d'écart un mille-pattes suivant sinueusement les contours du bas de la signature du scribe Aniceto May Tun en écho presque parfait à celui couvrant le dos du scribe singe sur un linteau palatial de Yaxchilan.

L'Aak'ab ts'iib rend manifeste le don scriptural, particulièrement mis en exergue par l'Almaj t'aan, ce message divin fondateur que seul le secrétaire et interprète de la Croix peut coucher par écrit et lire. L'Almaj t'aan partage avec d'autres écrits occultes des Mayas, ceux dits du Chilam Balam, la teneur d'un discours à la fois historique, politique et prophétique, ainsi que la déclinaison en copies partielles attachées chacune à des lieux signnifiants. Si l'on en sait très peu sur l'usage des manuscrits du Chilam Balam et sur la fonction prêtée aux passaǵes recopiés entre eux, les textes cruzo'ob donnent plus d'indications. Ici, la copie est clairement générative et créatrice. La «découverte», à savoir la reproduction manuscrite de nouveaux Almaj t'aan, semble avoir toujours accompagné la fondation de nouveaux centres cérémoniels.

Les Chilam Balam tout comme les carnets étudiés sont marqués d'hybridité interne, par les assemblages textuels et multitemporels qui les constituent, et de répétitions externes, par les relations de copie qu'ils déploient entre eux (et au-delà). Outre les réseaux de relations entre textes combinés (relations prêtées au moment de leurs écritures discontinues, de leurs lectures successives ou lors de leur redécouverte), ce qui compte du point de vue de l'efficacité de tels écrits est que ces assemblages rendent chaque carnet unique, ce que produisent également les variations dans la copie. En effet, ces répétitions ou copies, outre d'être souvent fraǵmentaires et objets de nouvelles combinaisons, ne sont jamais exactement identiques. On produit systémiquement du différent avec du même.

Les écrits cruzo'ob sont toujours liés à des performances orales, de divers types. Les Almaj t'aan étaient dictés nuitamment par une voix divine, puis rapportés cérémoniellement à un large auditoire. Les prophéties portées sur le papier et lues publiquement résonnent dans la tradition orale sous forme de citations, de narrations plus libres ainsi que de matrices énonciatives permettant de générer de nouvelles pré- ou postdictions (Vapnarsky 2017 : chap. x; 2018). Les carnets de prières semblent quant à eux instancier une relation entre écrit et parole plus contiguë ou 
pul thesensideduce s me nom nesicut Lac mut Sijti me kSi Cutcaseum mecoa gunlas tis pe the \& cor nibus 2 ses tis ta me osibuste mer tisisco ngisti me Vitam HmiSericor diam tribuistimihiet tisitititio tua CuSto dittit Spizitum emecum

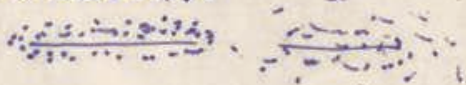
Anicto pray (c) sing fun :iniguitate sobser qatieris gomine Domine quis Susti nebit 1jial inios?

Reppofundis dama Uit ad te Domine Domine e Xau di tLo Sem meam fiat aure Stugeimtendentes im UTo cemde pre Catio

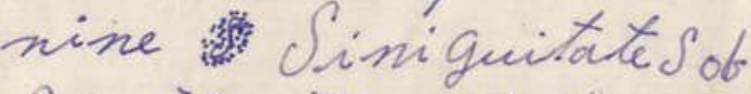
Sirltallenis Yomi

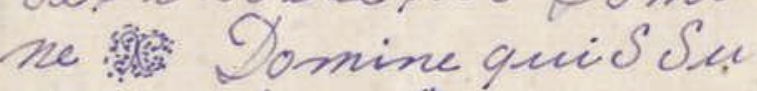
stinebit 


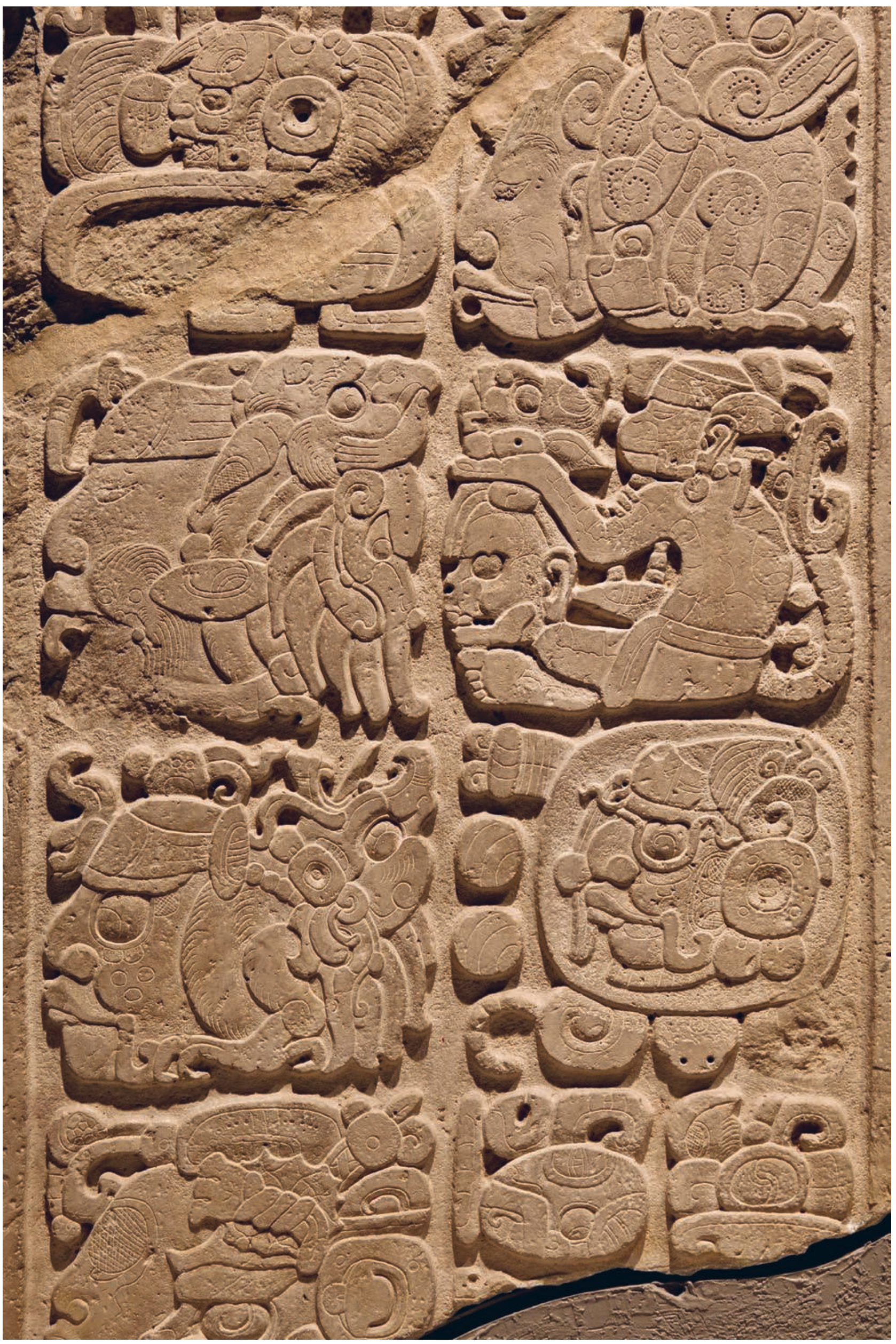

Glyphes maya sur le linteau 48, site Yaxchilan, Chiapas, 600-900 apr. J.-C. Mexico. Musée national d'Anthropologie, Mexico. Le glyphe de la deuxième ligne sur la colonne de droite représente un singe scribe avec un mille-pattes émergeant de son dos. Mostardi Photography / Alamy Stock Photo. 
littérale, a fortiori quand il s'agit de latin, lanǵue obscure qu'une larǵe majorité de prieurs ne comprend pas. Pourtant, c'est une dynamique de transformation continue qui, à l'inverse, caractérise ces carnets de prières, dans la tension et les va-et-vient entre mémorisation par l'écrit et performance des prières, oralisation et scripturalisation. La pratique de «copie» ne cesse ici de modifier les textes, non pas par «erreurs», mais parce qu'elle implique, pour être significative, l'intégration de changements singuliers et personnels.

Cela, dans un contexte culturel où, pour être efficaces, les paroles rituelles doivent nécessairement créer de la variation (Monod Becquelin et al. 2010).

De fait, en maya, l'acte de «copier» ne trouve d'autre nom que celui de «rénovation» ou de «renouvellement» (tu'umbenkúuns ${ }^{\mathbf{2 6}}$, littéralement «rendre nouveau»). C'est pourquoi la production d'une nouvelle version du même texte peut être réalisée avec le texte source sous les yeux, ou bien «en différé», des années après, cette fois à partir de la version orale altérée puisque opère la contrainte performative de variation. Dans le cas de nos amis prieurs, il s'agissait essentiellement de la mise à l'écrit d'évolutions dans le modus de l'oraison: vocalité et rythme. Dans le cas de Don Anis, s'ajoutaient clairement des reconfigurations matérielles liées à de nouveaux outils et supports et à de nouvelles performances. Ainsi, la «rénovation» des écrits et leur transformation textuelle et matérielle ne sont pas tant adaptation qu'individualisation et régénération. Le texte recopié ou à copier n'est pas conçu comme une matière fixe et stable. Si sa valeur mémorielle est indéniable, elle n'épuise en rien ses virtualités. Et si l'écriture dans son usage maya est restée jusque très récemment une écriture «attachée» (Déléage 2013), à savoir éminemment liée à certains genres de discours et certains types d'institutions, elle porte aussi en elle de devoir constamment se détacher, non pas de son contenu mais de sa propre forme. L'écrit sacré chez les Mayas n'est pas un objet intouchable. Au contraire, c'est une matière qui doit être l'objet de rénovations scripturales et d'oralisations renouvelées pour garder sa puissance, et la régénérescence inscrite dans sa nuit.
26. Mot formé à partir de la dérivation verbale causative (-kúuns) de l'adjectif tu'umben («nouveau»). 


\section{Bibliographie}

\section{Barbeau, Serge}

2017 Últimos testigos: The Last Rebellion of the Maya in Yucatán. Museum Fünf Kontinente, Hirmer

\section{Barrera Vásquez,} Alfredo et Rendón, Silvia 1978 [1948] El libro de los libros de Chilam Balam. México, Fondo de Cultura Económica.

\section{Becquey, Cédric}

2020 «De la nuit et de l'obscurité: une étude icono-épigraphique maya », Ateliers d'anthropologie 48 [en ligne], disponible sur: http://journals.openedition. oró/ateliers/13487 (consulté le 10 décembre 2020).

Bracamonte y Sosa, Pedro et Elmer Ek Ek 2014 Despues de 2012. Libro de la memoria y vaticinios mayas. México, Centro de Investigación y Estudios Superiores en Antropología Social.

\section{Bricker, Victoria $\mathbf{R}$.}

1981 The Indian Christ, the Indian King: The Historica Substrate of Maya Myth and Ritual. Austin, University of Texas Press.

\section{Bricker, Victoria Reifler} et Helga Maria Miram

2002 An Encounter of Two Worlds: The Book of Chilam Balam of Kaua, Nueva Orléans, Middle American Research Institute 68

\section{Caso Barrera, Laura}

2019 [2011] Chilam Balam of Ixil: Facsimile and Study of an Unpublished Maya Book. Leiden/Boston, Brill.

[Traduction d'El Chilam Balam de Ixil, Facsimilar y estudio de un libro maya inédito, Artes de México, Instituto Nacional de Antropología e Historia, México.]

\section{Ciura, Monika}

2019 « The Centipede in the Maya Art and Culture», Estudios Latinoamericanos 38: 47-77 [en ligne], disponible sur: https://estudioslatinoamericanos.pl/index.php/ estudios/article/view/

ES 383 Ciura/14 (consulté le 10 décembre 2020).

\section{Christensen, Mark Z.}

2016 The Teabo Manuscript: Maya Christian Copybooks, Chilam Balams, and Native Text Production in Yucatán. Austin, University of Texas Press.

\section{Cunill, Caroline}

À paraître Del ah ts'ib al escrivano: historia de una transición en el Yucatán del siólo XVI, in Carlos Conover Blancas et A. Rafael Flores
Hernández (dir.), El Área Maya en la Edad Moderna, México, UNAM. Instituto de Investióaciones Filológicas Centro de Estudios Mayas, p $12-45$.

\section{Déléage, Pierre}

2013 Inventer l'écriture: rituels prophétiques et chamaniques des Indiens d'Amérique du Nord, XVII - -XIX ${ }^{e}$ siècles. Paris, Les Belles Lettres.

Dumond, Don E.

1997 The Machete and the Cross: Campesino Rebellion in Yucatán. Lincoln, London, University of Nebraska Press

\section{Grupo Dzíbil}

1996 ms. Chilam Balam of Tuzik. Mexico, Sociedad Mexicana de Geografía y Estadística.

Hanks, William F.

2010 Converting Words: Maya in the Age of the Cross. Berkeley, University of California Press.

Harris, Charles H. et Sadler, Louis $\mathbf{R}$.

2003 The Archaeologist Was a Spy: Sylvanus G. Morley and The Office of Naval Intellioence Univeristy of New Mexico Press.

Ligorred Perramon, Francesc 2000 «El lenǵuaje de Zuyua y la resistencia literaria maya-yukateka colonial », Colonial Latin American Review 9 (1) : 49-61.

Liljefors Persson, Bodil

2000 The Legacy of the Jaguar Prophet: An exploration of Yucatec Maya Religion and Historiography. Lund, Lunds Universitet.

\section{Monod Becquelin} Aurore et al. (dir.)

2010 Figuras mayas de la diversidad. Mérida, UNAM.

Nelson, Reed

1964 The Caste War of Yucatan. Stanford, Stanford University Press.

Roys, Ralph L.

1973 [1934] The Book of Chilam Balam of Chumayel. Norman, University of Oklahoma Press.

Stephens, John Lloyd. 1848 Incidents of Travel in Central America, Chiapas, and Yucatan. New York, Harper \& Brothers for Henry Bill.

\section{Sullivan, Paul R.}

1984 «Contemporary Yucatec Maya Apocalyptic Prophecy: The ethnographic and historical context », thèse de doctorat en anthropologie. Baltimore, Johns Hopkins University. 1989 Unfinished Conversations: Mayas and Foreigners
Between Two Wars. Berkeley/ Los Angeles, University of California Press.

\section{1 (sous presse)}

«Los archiveros mayas de lo saǵrado », in Caroline Cunill et al. (dir.), Actores, redes y prácticas dialógicas

en la construcción y uso de los archivos en América Latina (siglos XVI-XXI). Mérida, Centro Peninsular en Humanidades y Ciencias Sociales, UNAM.

\section{Taube, Karl}

2003 «Maws of Heaven and Hell: The Symbolism of the Centipede and Serpent in Classic Maya Religion », in Andrés Ciudad Ruiz et al. (dir.), Antropología de la eternidad: la muerte en la cultura Maya. Madrid, Sociedad Española de Estudios Mayas: 404-442.

\section{Vapnarsky, Valentina}

1996 «The Voice of Prophecies Expressions and Visions of Time in Yucatec Maya ", in Ueli Hostettler (dir.), Los Mayas de Quintana Roo. Bern, Institut für Ethnologie: 13-39.

2017 «Les sens du temps : temporalités et temporalisations des paroles, expériences et mémoires mayas », mémoire d'habilitation à diriger des recherches. Paris, École des hautes études en sciences sociales.

2018 «De la politique à la grammaire. Recompositions prophétiques chez les Mayas Cruso'ob » in Chloé Andrieu et Sophie Houdart (dir.), La Composition du temps : prédictions, événements, narrations, actes du $14^{\mathrm{e}}$ Colloque de la Maison archéologie et ethnologie René-Ginouvès (Nantere 7-9 juin 2017). Paris, Éditions de Boccard: 203-215.

\section{Villa Rojas, Alfonso}

1987 [1945] Los elegidos de Dios: etnografía de los mayas de Quintana Roo. México, Instituto Nacional Indigenista. [Traduction et nouvelle édition de The Maya of East central Quintana Roo, Washington D.C. Carneǵie Institution of Washington, 1945.]

1977 «Los Mayas del actual territorio de Quintana Roo», in Carlos A Eschánove Trujillo, Enciclopedia Yucatanense 6: 31-62. México, Ed. Oficial del Gobierno de Yucatán.

1984 «Valor histórico y etnográfico de los libros de Chilam Balam », Anales de antropología 21, UNAM.

\section{Whalen, Gretchen}

2003 «An Annotated Translation of a Colonial Yucatec Manuscript: On Religious and Cosmological Topics by a Native Author», Report,

Foundation for the Advancement of Mesoamerican Studies [en liǵne], disponible sur: www.famsi.org/reports /01017/ (consulté le 10 décembre 2020).

\section{Ci-contre et ouverture}

Pages d'un des carnets de prière mayas étudiés [c2,10-11] région cruzo'ob, Mexique début du xxe siècle (détail). 
